\title{
Towards a 3D passive dynamic walker to study ankle and toe functions during walking motion
}

DOI:

10.1016/j.robot.2019.02.010

\section{Document Version}

Accepted author manuscript

Link to publication record in Manchester Research Explorer

\section{Citation for published version (APA):}

Wang, K., Tobajas, P. T., Liu, J., Geng, T., Qian, Z., \& Ren, L. (2019). Towards a 3D passive dynamic walker to study ankle and toe functions during walking motion. Robotics and Autonomous Systems.

https://doi.org/10.1016/j.robot.2019.02.010

\section{Published in:}

Robotics and Autonomous Systems

\section{Citing this paper}

Please note that where the full-text provided on Manchester Research Explorer is the Author Accepted Manuscript or Proof version this may differ from the final Published version. If citing, it is advised that you check and use the publisher's definitive version.

\section{General rights}

Copyright and moral rights for the publications made accessible in the Research Explorer are retained by the authors and/or other copyright owners and it is a condition of accessing publications that users recognise and abide by the legal requirements associated with these rights.

\section{Takedown policy}

If you believe that this document breaches copyright please refer to the University of Manchester's Takedown Procedures [http://man.ac.uk/04Y6Bo] or contact uml.scholarlycommunications@manchester.ac.uk providing relevant details, so we can investigate your claim.

\section{OPEN ACCESS}




\section{Accepted Manuscript}

Towards a 3D passive dynamic walker to study ankle and toe functions during walking motion

Kunyang Wang, Pablo Tena Tobajas, Jing Liu, Tao Geng, Zhihui Qian, Lei Ren

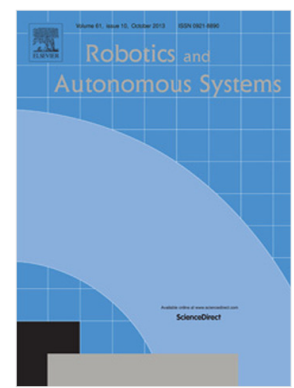

PII:

S0921-8890(18)30685-7

DOI: https://doi.org/10.1016/j.robot.2019.02.010

Reference: ROBOT 3171

To appear in: Robotics and Autonomous Systems

Received date : 27 August 2018

Revised date: 2 January 2019

Accepted date: 13 February 2019

Please cite this article as: K. Wang, P.T. Tobajas, J. Liu et al., Towards a 3D passive dynamic walker to study ankle and toe functions during walking motion, Robotics and Autonomous Systems (2019), https://doi.org/10.1016/j.robot.2019.02.010

This is a PDF file of an unedited manuscript that has been accepted for publication. As a service to our customers we are providing this early version of the manuscript. The manuscript will undergo copyediting, typesetting, and review of the resulting proof before it is published in its final form. Please note that during the production process errors may be discovered which could affect the content, and all legal disclaimers that apply to the journal pertain. 


\title{
Towards a 3D passive dynamic walker to study ank'e and toe functions during walking motion
}

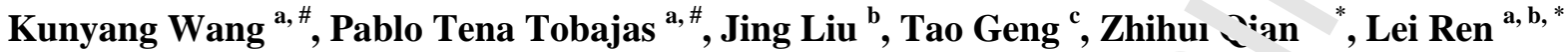 \\ ${ }^{a}$ School of Mechanical, Aerospace and Civil Engineering, University of Manchester, M13 9PL Manct ster, ${ }^{\mathrm{TW}}$ \\ ${ }^{b}$ Key Laboratory of Bionic Engineering, Ministry of Education, Jilin University, 130025 Changchur Thin \\ ${ }^{\circ}$ School of Information Science and Engineering, Harbin Institute of Technology (Weihai), 264209 Welm China \\ \# These authors contributed equally to this work. \\ * Correspondence to:
}

Lei Ren

C13, Pariser Building

School of Mechanical, Aerospace and Civil Engineering

University of Manchester

Manchester, M13 9PL, UK

Tel. +44 (0) 1613064251

E-mail: lei.ren@manchester.ac.uk

Zhihui Qian

Key Laboratory of Bionic Engineering

Jilin University

Changchun, P.R. China

130022

Tel. +86 (0) 43185095760

Email: zhqian@jlu.edu.cn

\section{Declarations of interest: none}

\begin{abstract}
The ankle-foot complex in the $\mathrm{h} r \ldots$... $\eta$ body is one of the major determinants in normal human walking. Most of the research study ankle and foot $r$ otio' by observing people as they move, measuring desired kinematic and kinetic data indoor or outdoor, and numt. 'al simulations in computer. However, very few studies are able to explore the fundamental mechanical pr ‘cip' ss underlying human musculoskeletal system. In this paper, we developed a threedimension (3D) passive $\mathrm{d},{ }^{\prime} \cdot \mathrm{r}, \mathrm{c} \mathrm{W}^{\prime}$ k ker with flat feet, toes and ankle springs to investigate the impact of the ankle and toe stiffness in the ${ }^{\prime \prime}$ king. "cion. The results suggest that the ankle springs have a main impact on the walking motion, wher the an rior spring, over any other position, plays a main role in providing sagittal stability. The springs from the $\mathrm{s}$. oittal $\mathrm{p}$ ine control the pitch angle of the robot which impacts on its velocity and step length. The stability got $w$, we aluns with the step length and velocity increasing especially when the step length overcame $8 \mathrm{~cm}$. The fact th $\mathrm{t}$ the be : configuration of the ankle joint has stiffer stiffness in the sagittal plane than the coronal plane complies in ature /ith humans where Tibialis Anterior, Soleus and Gastrocnemius muscles are much stronger than thar muscles around the ankle. Furthermore, it can be stated that the medial toe plays a more important role ' $\mathrm{a}$. the lateral one, as blocking the medial toe with the stiffest joint (rigid joint) has a negative effect on walking motic In conclusion, we show that the ankle stiffness of the robot in anterior-posterior position should be higher than that in medial-lateral position and the stiffness in any position should exceed a minimum level to maintain walking stability. Also, adding toes (medial one should be softer than the lateral) in the foot of the robot may benefit biped locomotion especially when taking longer step length.
\end{abstract}

Keywords: biologically inspired robot, passive walker, biomechanics, ankle, toe 


\section{Introduction}

Human walking is a dynamic, partly self-stabilizing process relying on the interaction of the biomechanical design, i.e., the musculoskeletal system, with its surroundings. Nature has created successful solutions adapted to different uses and environments that develop gr at capabilities with apparent simplicity. The most conventional methods of studying human walking a e v verving people as they move [1-3], measuring desired data by electrical devices during experimen ${ }^{+\sim}$ nn a real person (e.g., motion capture [4-6], surface electromyography [7-11], medical imaging (12-4]), and numerical simulation [15-19] in computer (e.g., rigid body modelling and continuum mec anics modelling), etc. However, very few studies are able to explore the fundamental mechanical prin : nles underlying human musculoskeletal system as the results could not be validated in a real perss 1.

Indeed, biologically inspired bipedal robots can provide an at $t_{1}$ - ti / e alternative approach to investigate the what-if questions in human movement by examinir, the 11, portant components in a scientific cycle of producing hypotheses, conceptions and assumptic $1 \mathrm{~s}$, test $1 \mathrm{~g}$ them in experiments and adjusting them properly towards new theories. Repeatable, parameter. ${ }^{\circ}$ a $\mathrm{e}^{-}$.periments can be conducted in the robot model by programming. Relevant variables and para' teter ' mmeasurable in human trials can be monitored by implementing multiple sensors (e.g., internal for s) in hem. The morphology of robots can be changed in a systematic way to perform different tasks. They un perform movements that could be dangerous or even unreasonable for human, exploring the optir ization of human movements (e.g., motions, exercises or sports that are helpful for human $\mathrm{h} \sim a l t h$, $\mathrm{T}^{1}$ \& past decades, especially after ZMP criterion $[20,21]$ and its relation to human walking stability 'eing proposed in 1969, have seen great progress and advance in bio-inspired bipedal robots tha ${ }^{2} 1 \mathrm{~m}$ at complexities such as ASIMO [22], NAO $[23,24]$, Petman [25], Atlas [26], etc. Yet, most of those ru ots are incomparable with humans from a biomechanical point of view. Recently, passive dyn، ni walkers (e.g., McGeer biped [27], MIT biped [28], Denise [29] and Cornell biped [30]) whic! hase heir locomotion on natural dynamics solely are proposed as the new design and control paradigm 4 , 1h estigate human biology, especially biomechanics and neuroscience.

Human ankle-foot complex plays a determinan. "ole in human locomotion as it is the only part which contacts with the environment. Underst-- - ing the natural regulation of ankle-foot functions during walking motion is necessary to explor, how umans interact with environments, and also provide a foundation design principle for the deve, $\mathrm{mf}^{\mathrm{t}}$ t of humanoid robots. In literature, ankle function has been studied extensively in various $r$ ond ions. System identification analyses [31-34] are widely used to

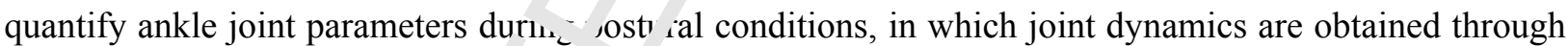
the analysis of the relation betwe in the $1_{1}$ ut (position, torque and EMGs signal) and output (torque and position) records. Wearable an le $\mathrm{l}^{\prime}$ 'nts [35] or specific instrumented platforms [36] (e.g., 3D motion capture system[37]) have rec ... $v$ been developed to estimate joint functions during walking. However, those studies have not chare teri ed the ankle-foot complex functions throughout the complete gait cycle, and our knowledge of ank' $\mathrm{e}$ an $\mathrm{u}^{\prime}$ 'e regulations during dynamic tasks such as walking is still incomplete.

Recently, some con ent' nal biped robots equipped with new design of ankle or toe joints has come out to study the function - hu ran foot in gait or human-like biped locomotion (particularly walking or running). Otani et a' [38-49] ueveloped a biped robot with leaf springs (acted like torsion spring in the sagittal plane) in th ? ankle to explore human running. It can perform active push-off during running driven by active ${ }^{1}$ nee, $\cdots$, and passive ankle. In 2006, WABIAN-2R robot [41] was built by Waseda University to a hieve uman-like walking. A passive toe that can rotate freely in the sagittal plane is added in the foo, hut $t^{\prime}$.e main contribute to the ability of mimicking the knee stretched, heel-contact and toe-off mot: ... - f hiıman walking are the powered ankle, knee and hip joints. There is no further study on the function $c^{*}$ ae toe. WABIAN-2R evolved in 2010, where a new foot mechanism containing medial longitudinal arci and two toes [42] has been applied to figure out the function of the arch structure in human foot. The motion of the medial toe is limited by connecting a stainless wire to the arch and the lateral toe can perform free rotation in the sagittal plane. Hashimoto et al [42] found that the changes of the elasticity of the medial longitudinal arch could reduce foot-landing force during walking, but the toes function has still not been answered. Moreover, HRP-2LT [43] and HRP-4C [44, 45] used passive or active toes to imitate human walking pattern. From the standpoint of engineering, these robots with special design in the ankle or toes can imitate human-like locomotion gait (e.g., toe-off) using high- 
torque motors mounted in the joints and sophisticated control algorithm. However, they have neither quantitatively evaluated how the mechanical impedance of ankle or toes affects biped walking or running from a biomechanical point of view, nor considered the medial-lateral (i.e., in the coronal plane) elasticity or compliance of the ankle.

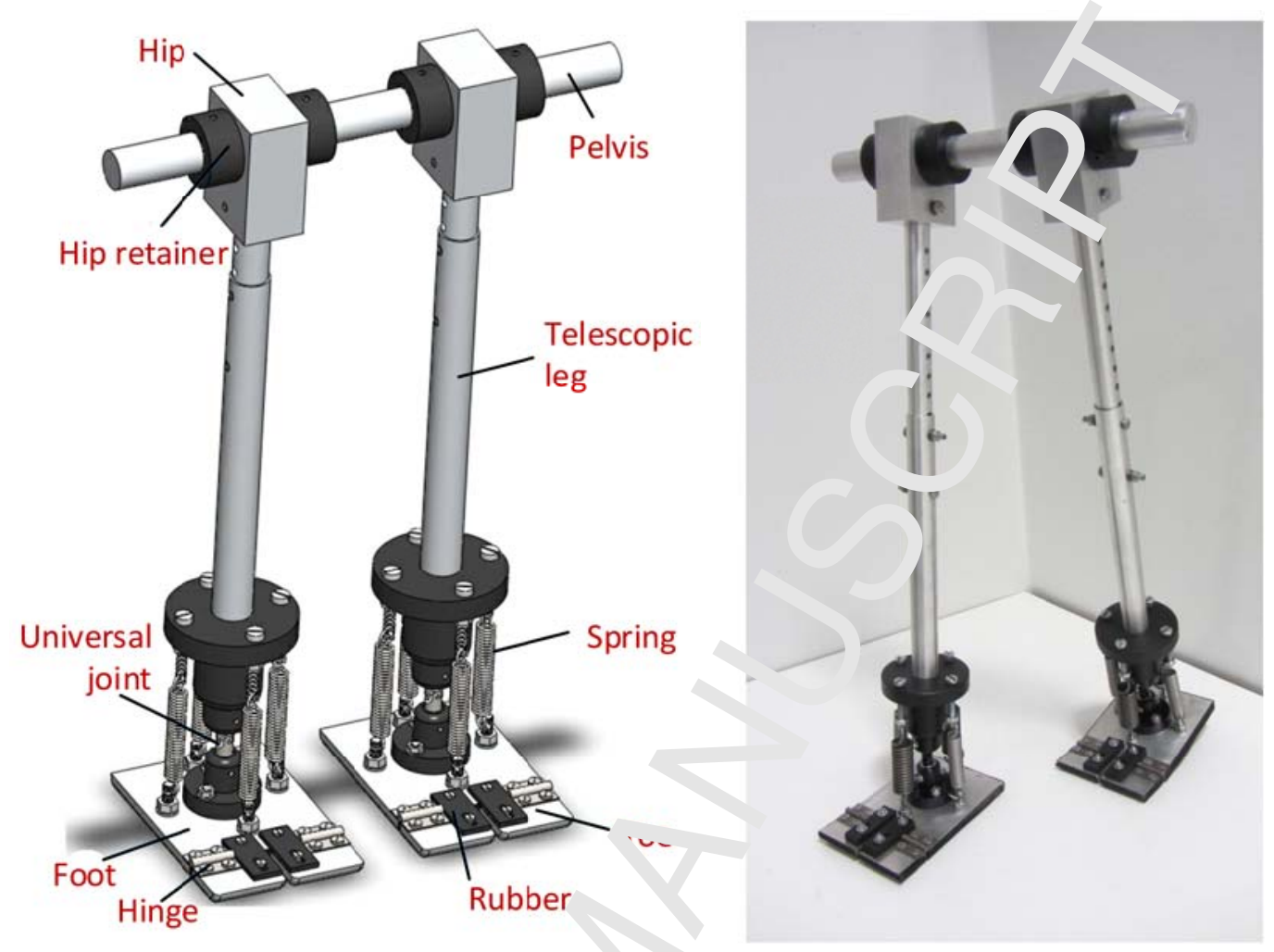

Figure 1. 3D Bipedal rot …th i at foot, toes and ankle springs.

In this paper, we developed a 3D passive dynamic walker (Figure 1) to be used as a platform to evaluate the ankle and the toe functions, espu ially the stiffness, during walking motion. The research focused on the foot, ankle and toes desis tryins to mimic human walking and understand the advantages of human ankle-toes structure. Previs is work is the literature developed bipedal robots with flat feet and ankle springs (e.g., the walker fror $\mathrm{K} f \mathrm{o}$ U iversity [46, 47]) lack of further research on the effect of each spring position in the walki $\mathrm{g}$ mu. ${ }^{\prime}$. They were limited to set the same type of spring in all the ankle positions, instead of cor $\jmath_{1}$ ing different stiffness springs at the same time and analyzing their impact on the motion and stability of $u_{1}$ r robot. Section 2 describes the basic biomechanics of the anklefoot-toe complex in human sod during normal walking. Section 3 presents the design concept of the passive dynamic walker. Sec. " n 4 outlines a series of walking tests to investigate the effects of each ankle spring position (an erio", posierior, lateral and medial) in the walking motion, to analyze the impact of the ankle springs w. 'ir the sagittal and coronal planes to the roll, pitch and the forward swinging motions of the leg, to ddy $\iota_{\text {. }}$ toes effect on the stability in function of their joint stiffness at medial and lateral position. Ser ion 5, ve discuss the results and the limitations, conclude this paper, and suggest new guidelines and $a$, rigns o be followed in the future research.

\section{Biomecl. ?ni's}

The prim ry cask of the ankle-foot complex is to provide an adaptable, stable, and efficient interface between the boi $r$ and the ground during human locomotion. This task requires the ankle and the foot to be sufficiently pliable during early stance phase to conform to various surface terrain, to absorb and translate energy while maintaining whole-body stability, and to rapidly achieve sufficient rigidity during late stance phase to propel the body forward using the rigid lever of the longitudinal arch [48].

The key movement of the ankle joint complex are dorsiflexion and plantarflexion, occurring in the sagittal plane, and inversion/eversion occurring in the coronal (frontal) plane (see Figure 2). Due to the projection of the foot anteriorly from the coronal plane of the body, the terminology describing motion of 
the foot differs in several important ways from standard descriptions of motion in other areas of the body [48]. First, the inversion and eversion represent motion in the coronal plane about an anterior/posterior axis (known as abduction/adduction elsewhere in the body), which occur primarily at the subtalar joint and can be demonstrated by moving the plantar surface of the foot to face medially (inversion) or laterally (eversion). Second, flexion/extension of the foot is termed dorsiflexilo . and plantarflexion respectively. This motion occurs around a medial/lateral axis in the sagittal plan', 1.c. vrimarily at the talocrural joint.

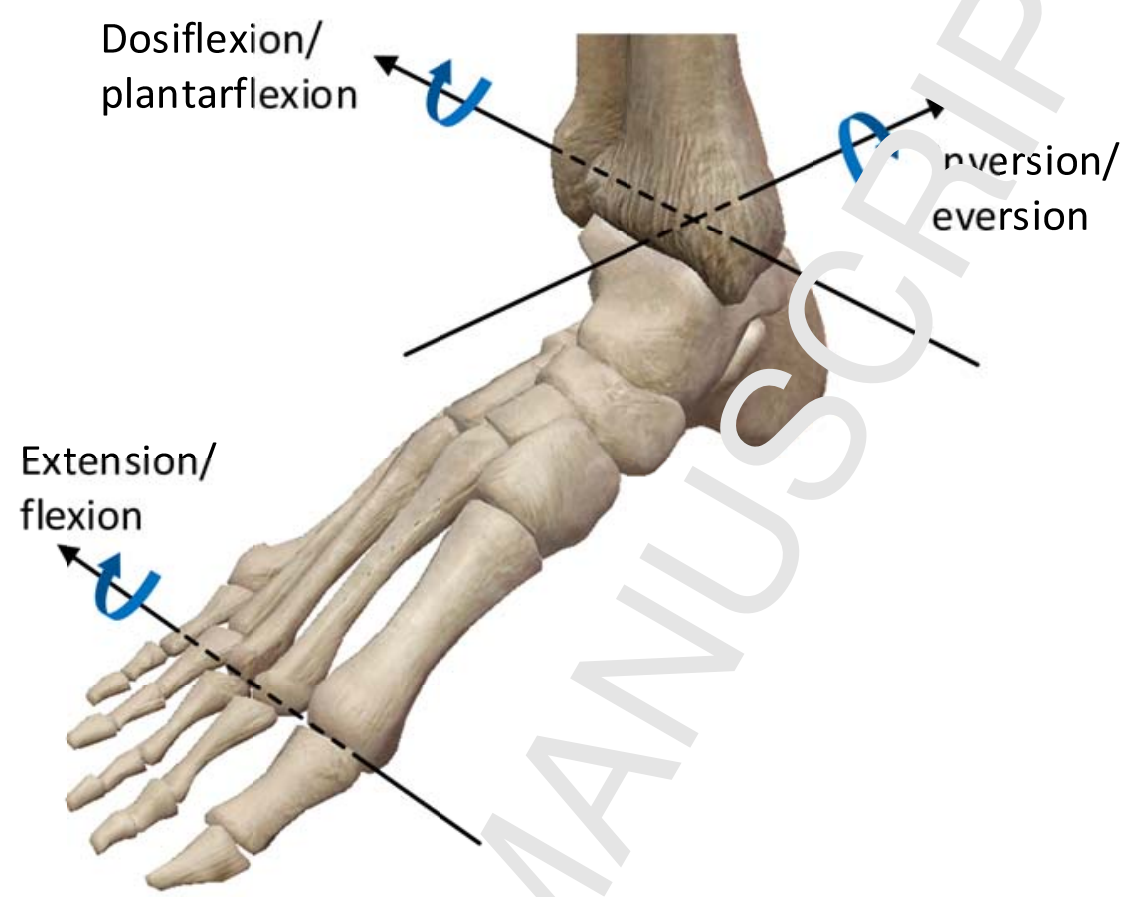

Figure 2. Motions in the ankle and toe joints. The key movements of the ankle joint complex are dorsiflexion/plantarflexion occurring in the sagittal plane and inversion/eversion in the Coronal (Frontal) plane. The major movements of toe joint are exter sion a 'd flexion.

At initial contact during human walk ${ }_{1 .}$. he talocrural joint is neutral or slightly plantarflexed. It plantarflexes more before loading resr unse as the foot is lowered to the supporting surface, then dorsiflexes before midstance as the 1 ver ' $\mathrm{g}$ g rotates medially and anteriorly over the supporting foot. During terminal

stance and pre-swing, the taloc.ural, int has large plantarflexion as the body weight is transferred onto the

contralateral limb, and it $\mathrm{r}_{\text {. nid }}{ }_{y}$ dorsiflexes to the neutral position immediately following toe-off, to attain toe clearance and th in may nlantarflex slightly in preparation for next cycle.

The subtalar joint $r$ ate in the whole walking cycle, which influences the weight-bearing alignment of the entire lower body 4 rin, stance. Similar to the talocrural joint, the motion range of the subtalar joint is small compa ed $\mathrm{wi}^{\text {th }}$ the hip or knee, however it is the indispensable motion that permits humans to adapt to various s rfaces It functions as a mitered hinge to transmit external/internal rotation from the tibia to eversior"...versivi about the foot, and vice versa. Since body weight is transferred onto the supporting foo during human walking, eversion of the subtalar joint, as a normal passive response to initial contact $W_{1}{ }^{h}$ the neel, is one of the motions that absorb shock and prevent injuries. It unlocks the midtarsal jc 's . nroduce a relatively flexible forefoot. Meanwhile, subtalar inversion helps to induce foot stability c ' ing single limb stance. Maximal subtalar eversion and inversion occur at foot flat in early stance phase ano it toe-off respectively.

The key movement of the toe joints during walking are flexion and extension (see Figure 2). The toes are off the ground in extension at initial contact, then flex to neutral position from forefoot contact with the ground until midstance. During terminal stance, they extend once again while remaining in contact with the ground, and a maximum of toe extension is reached during pre-swing. The toes flex slightly but remain extension during swing. In the end, a minimal increase in toe extension comes up in preparation for initial contact of next walking cycle. Little or no toe flexion occurs during human walking. 
As not all structures and features during human locomotion are dispensable for robot locomotion, only replicating the key relevant features to design a bio-inspired bipedal robot is requisite. Inspired from the basic biomechanics of the ankle-foot complex especially the ankle and toe motion during human walking, we developed a 3D passive dynamic walker with flat feet, toes and ankle spring system.

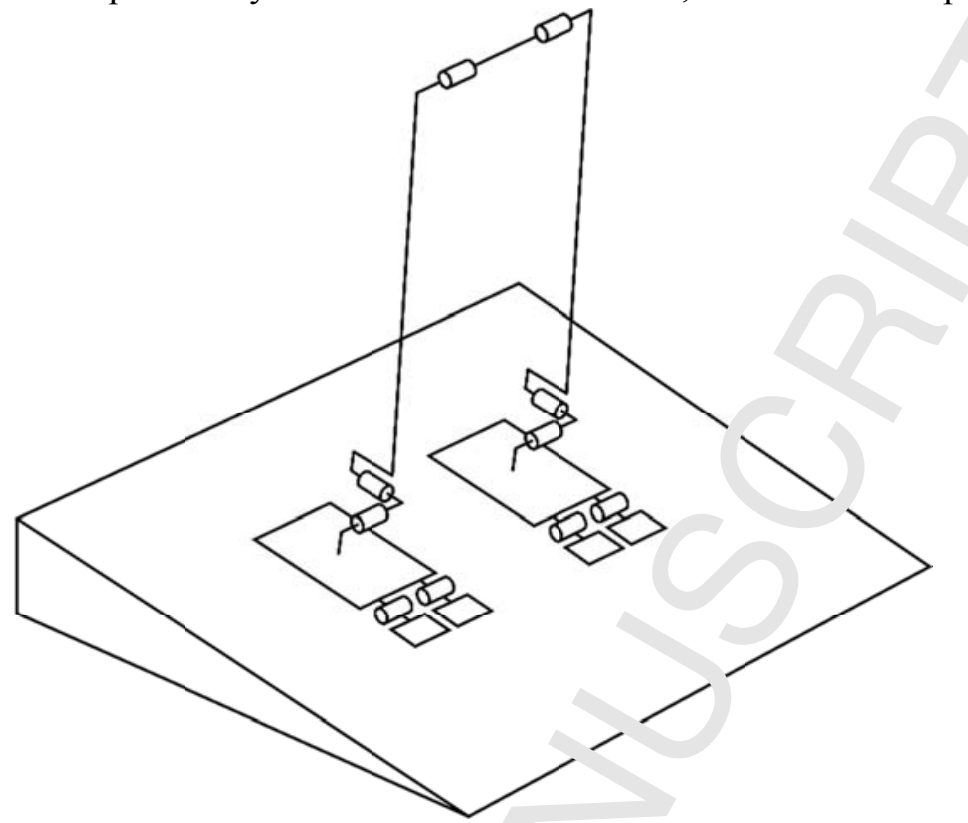

Figure 3. The system configuration of the bipedal walker. It $~-n s i s t s$ of a pelvis, two straight legs, two feet, and four toes with 10 DoFs.

\section{Robot Design}

The walker (see Figure 3) consists of four 1 . 'in segments (foot, toe, extensible leg and pelvis) and three joints (ankle, toe and hip joints). Each foot includes two toes fixed by steel hinges and plates made of rubber that act as springs forcing the toes - return to the straight position at each step. Each ankle includes a universal joint, four springs a. $t$ two components made of Delrin connecting the foot and the leg. The telescopic leg links the ank ${ }^{1}$; with tw hip, whose extensible feature allows the robot to change the step length, velocity and dynam ' $\mathrm{c}$ the walking motion.

The robot weights $2.47 \mathrm{~kg}$, ald its $\sim$ dl height goes from 422.81 to $617.81 \mathrm{~mm}$ depending on the setting of its telescopic legs, me s.s. $-d$ from the highest point at the hip to the ground. The dimensions are based on humans. It is well kn ${ }^{n}$ wn that ine average human height for men is $176.3 \mathrm{~cm}$ which supposes an average leg length of approy mat ly $90 \mathrm{~cm}$ and foot size $25 \mathrm{~cm}$ leading to a ratio between the leg and foot size of 3.5 [1]. Hence, the ht, nt-foot ratio of the robot was designed to consider this value among its different height settings, gc ing trom 3.2 to 4.7. However, for passive dynamic walkers, achieving walking stability using ${ }^{\prime}{ }^{\prime} n$ an $r^{\prime}$ 'mensions is quite difficult as they do not have muscles, tendons or any control systems that wn prov. e active stability at every instant. Nevertheless, the design was developed including the extren e positı ns to test its limit capabilities.

Also, the weight 'istri', ution is one of the most important aspects. Based on the Matlab simulation, the CoM (centr : of m 'ss) of the robot should be placed at high point to enhance the roll and walking motion down th. slope due to gravity. The size of each component as well as the material type plays a key role in the mass ustribution and in the final position of the CoM.

The foot is ane of aluminium with sufficient strength, weight and high reliability. Each foot was drilled to fix th ankle springs, toes and legs support, and chamfers were fabricated in the edges of the heel, foot sides and toes to improve the contact condition which could enhance the stability and reduce bouncing and perturbations created by the impacts. The leg made of aluminium is adjustable on length by setting the upper part inside the lower part and fixing them together with bolts. The objective of this feature is to change the step length and dynamics in order to achieve a successful configuration as well as allowing the study of their impact on the walking motion. The primary function of the pelvis and the hip joint is to support the weight of the upper body during walking. Since the robot without the upper body 
was developed to study the ankle-foot complex of human body, the pelvis was simplified to an aluminium round bar, and the hip joint to a hinge joint with one DoF rather than a ball-and-socket joint with three DoFs minimizing the instability during walking.

Ankle joint. As the main motion in the ankle joint during $\mathrm{hr}$ nan walking are dorsiflexion/plantarflexion and inversion/eversion, it could be replaced using a un arsal joint which permits movements in the sagittal and the coronal plane.

Ankle Springs System. The ankle spring system controls roll and pitch mu in of the robot at any instant allowing the legs to swing forward and sideward within a wide range $c^{-}$' $n g$ ens. Stiffness, initial length and elongation are the main factors that affect walking motion. $\mathrm{H}^{\prime}$ ma ${ }^{\prime}$ muscles do not apply the same forces independently of the walking conditions, but adapt permane. ${ }^{-1}{ }{ }_{i}{ }_{1}$ function of factors such as ground slope, friction coefficient of the surface, weight distribution, act:-ity a eloped by the human, etc. The spring system acts in the same way and should adopt accl ate ch nges to set the successful adjustments and combinations that achieves walking stability for deter rined onditions.

The spring system (Figure $4 \mathrm{~b}$ ) was designed to reach a wide range of possible initial spring elongations so that the robot could easily and accurately adapt to tis . erer . walking conditions. They were selected based on their force-elongation rate to cover the whı: ro' .. spectrum of settings. The initial spring length was $63.5 \mathrm{~mm}$ and their rates went from $1950 \mathrm{~N} / \mathrm{m}$ to $6,70 \mathrm{~N} / \mathrm{m}$. Three types of springs with different stiffness (soft, medium and hard) were combinet in ' no 'tions (anterior, posterior, lateral and medial) to study the ankle spring functions (Figure 4a).

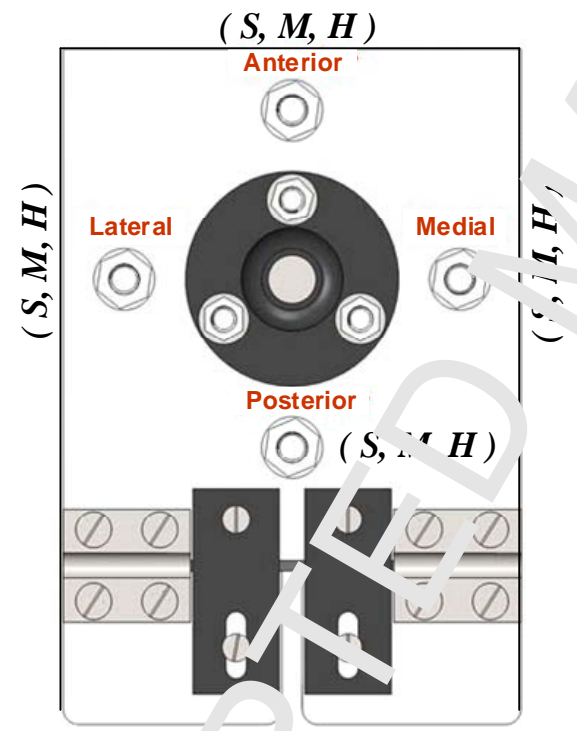

a)

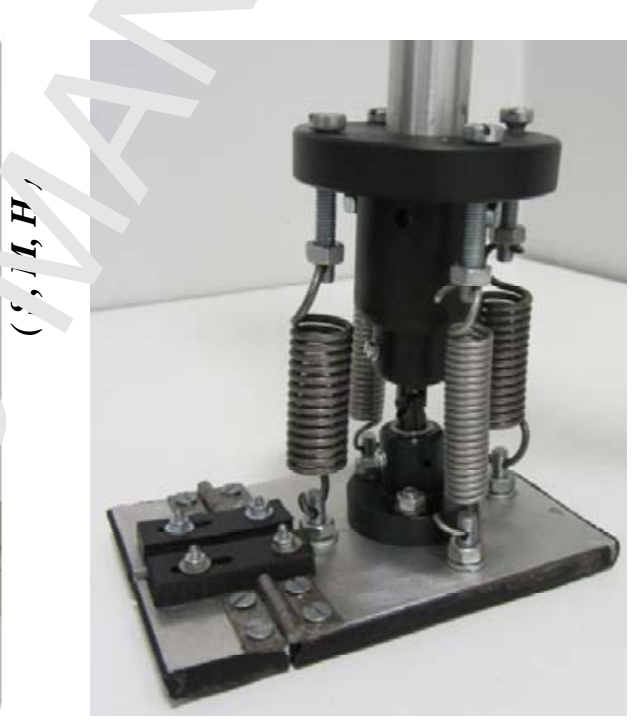

(b)

Figure 4. Configuration $c$. the ankle spring system. (a) Schematic of the right foot ankle spring settings with anterior-posterior ankle _.ri gs $\ldots$ sagittal plane and lateral-medial ankle springs in coronal plane. Three springs with different ' ' ffness e combined in 4 positions, where ' $\mathrm{S}$ ' is soft spring $(1950 \mathrm{~N} / \mathrm{m})$, ' $\mathrm{M}$ ' medium spring $(3260 \mathrm{~N} / \mathrm{m})$ al $\mathrm{d}$ ' $\mathrm{H}$ ' hc $\cdot d$ spring $(6670 \mathrm{~N} / \mathrm{m})$. (b) Real ankle joint in standing position. 


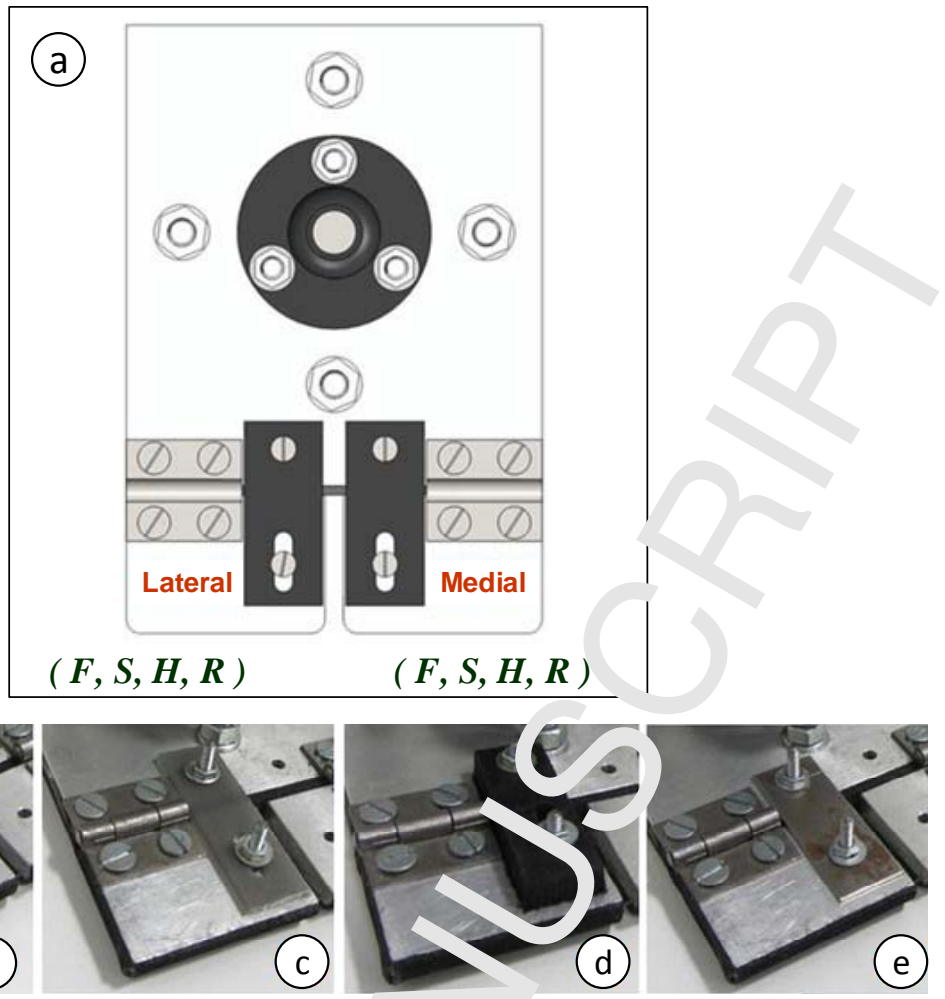

Figure 5. Configuration of the toes. (a) Schematic of the laı 91 and medial toes settings in the right foot. 4 different toe springs with different stiffness are combil $u$. ? nositions, where ' $F$ ' means free moving without spring (b), 'S' means soft spring (c), 'H' means hard sprı ${ }^{\circ}$ (d), and ' $R$ ' means rigid joint without motion fixed with a steel plate (e).

Toes. Two toes pursue to improve the. .11 in. stability by increasing adaptability and contact duration between the foot and the ramp. The toc. were fixed to the foot by steel hinges placed in the lateral exterior edges of the feet (see Figure 5), which could create sufficient clearance for the elastic rubbers. The elastic rubbers drove the to $\mathrm{s}$ to $\mathrm{re}$ irn to the straight position. The elastic rubbers allowed a great variety of strength by changing its $\iota_{1} \cdot k n e s$ or material. In order to validate whether the toes have a real impact on the walking motion of his bipeual robot, the tests include 4 types of toe setups (see Figure 5) with different stiffness.

\section{Tests and Results}

Although numerical si: '1la' on has successfully facilitated the design of 2D passive walkers, 3D passive-dynamic simulati $\mathrm{n}$ is $\mathrm{H}^{+}{ }^{+}$as accurate and useful as that of $2 \mathrm{D}$ bipeds. There are many uncertain effects in 3D analysis e.s., collision, rolling, friction and scrubbing torque, that are difficult to characterize and to deter. ne ne importance [49]. Simulation sometimes leads to different results in physical robot, such $t$ s the Delft robot [50] whose swift swing-leg motion cannot increase lateral stability as showed in simu ations. Presumably, the instability of numerically predicted 3D biped motion in simulation render physicul realization. Therefore, the robot is studied by a trial and error process testing all the different configu ations and analyzing their impact on a real experiment.

Two main tc. ${ }^{+} \mathrm{s}$ are pursued in this paper, the ankle stiffness effect based on the spring system and the toes effect $\ldots$.... ralking motion. They are studied separately: the ankle stiffness is studied by setting all the different $s_{1} r$ ng configurations, then recording and analyzing the results obtained; the toes stiffness is evaluated based on the best configuration obtained from the ankle spring tests to investigate the real effect of the toes on walking motion. Besides, more tests and configurations have been conducted to study the effect of some specific variables in the walking motion and improve the design for future research.

The robot was tested at two different walking conditions: short step and long step. In general, at the short step condition, the step length is below $7 \mathrm{~cm}$ and the robot tends to walk slowly. Whereas during the long step condition, the step length is above $7 \mathrm{~cm}$ and the robot moves fast. The different walking 
conditions were achieved by initially launching the robot using different methods. The initial launching condition of the robot defines the walking type of the trial.

During tests, the robot motion was recorded by using a video camera at $60 \mathrm{~Hz}$. The number of steps, distance travelled, the total time duration from launching to failure, average velocity and average step length were obtained by analyzing the video frames. The distance travelled was mear ured from the toes at the initial position to the toes at the last successful step. The total time duration was acquired directly from the video frames. The average step length was calculated by dividing the number of steps, and the average velocity was calculated by dividing the dist nce ravelled by the total time duration. Unstable motions of the last steps, mainly in the stopping seq'ence, i e. very short step of less than $1 \mathrm{~cm}$ length, or in the falling sequence, i.e. very long last step just be $e^{-a}$ losing balance, were all excluded when analyzing the video data.

\subsection{Variables that affect the walking motion}

The robot walking is influenced by various variables that chan $:$ its mo vements, velocity, stability, and ultimately its success. It can be assumed as a problem to be iver for each set of determined variables. The variables can be grouped in 3 sets: external, int nal related to the walking motion. External variables are derived from outer elements to the robot, $c_{-2}$, the ,lope, friction or damping of the ramp. Internal variables are derived from the robot settings, - $\sigma_{\text {., }}$ ue ankle springs, legs length, hip masses. Variables related to the walking motion change the soluti $\mathrm{n}$ to the problem depending on the motion pursued, e.g., walking at a higher velocity and taki $\bullet o$ lo. ${ }_{-}$er steps leads to a different setting.

\subsection{Preliminary test}

Preliminary tests were conducted to determine the op: 'nal leg length and better ground surface enabling the robot walking stably. The robot was tes ${ }^{\prime} d+\mu$ uach of 13 different leg lengths ranging from $422.81 \mathrm{~mm}$ to $617.81 \mathrm{~mm}$. It was found that leg '^ngth has significant impact of walking dynamics as it affects the leg mass distributions and also the joint ry es. The optimal leg length was found at $549 \mathrm{~mm}$, with which the robot achieved most stable $\mathrm{w} \cdot \cdots \operatorname{mo}$, otions for both short and long steps. This optimal length was used in the tests investigating the effec of the stiffness of the ankle and toes. Additionally, in the initial tests, the ground friction of the ramp was insufficient, and the robot slipped during walking especially at long steps. Thus, we sr lded t e ramp surface to increase the ground friction, and successfully solved the slipping problem.

Also, foot soles were designed tr red ce the vibrations generated by heelstrike with the ramp. Rubber

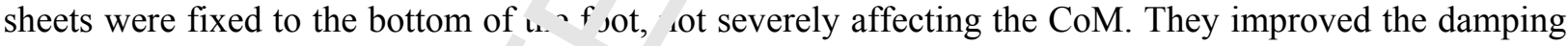
capabilities by absorbing the ener sy rele $a_{n}$ d during heelstrike that produced disturbances during walking.

\subsection{Ankle test}

The ankle spring was te ted ,etting all the spring combinations to find out which performs the most stable walking motion for she and long steps separately. The test was developed based on the best configuration attained $\mathrm{d} \mathrm{r}$ ing preliminary test, and the toes were equipped with the stiffest rubber.

\subsubsection{Ankle spring $s^{\circ}, \ldots$.ess.}

Three types of ( Ifferent springs were used to test the robot, resulting in 81 configurations to be tested with two different $m_{1}+$ inon , raising the total of 162 testing combinations. For each combination it was launched sever . time but only taking the first 5 successful ones, and the average of each variable was calculated to be compar $2 \mathrm{~d}$ with the rest. The measurements for all the launches and configurations can be found in Sunnlemerualy Table S1.

The stan 'arc' aeviation of distance travelled for short (Figure 6b) and long (Figure 6d) step walking were computea to show the data variation of each test, which indicates that the following analysis are reasonable as most of deviation value are small compared with the average value of distance travelled. 


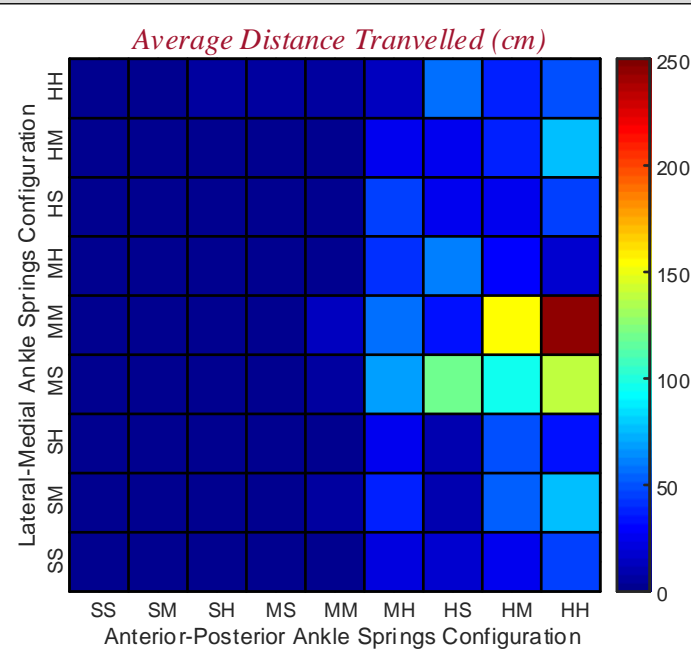

(a)

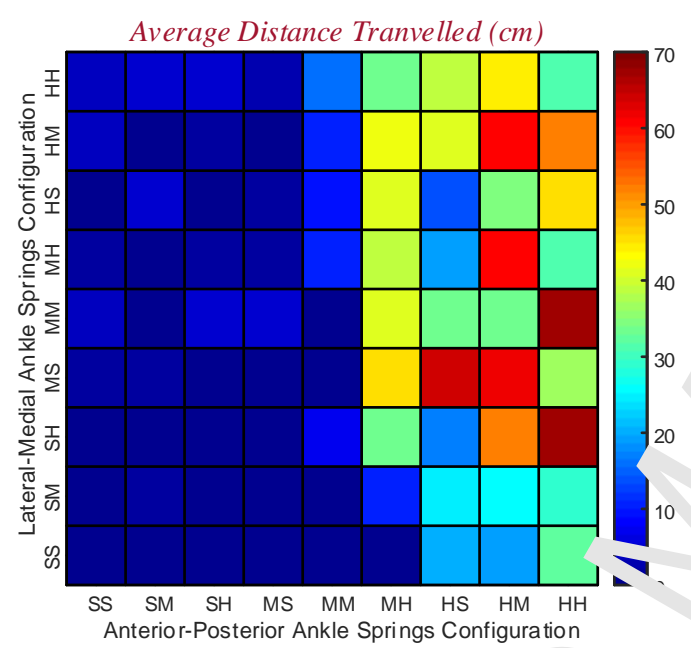

(c)

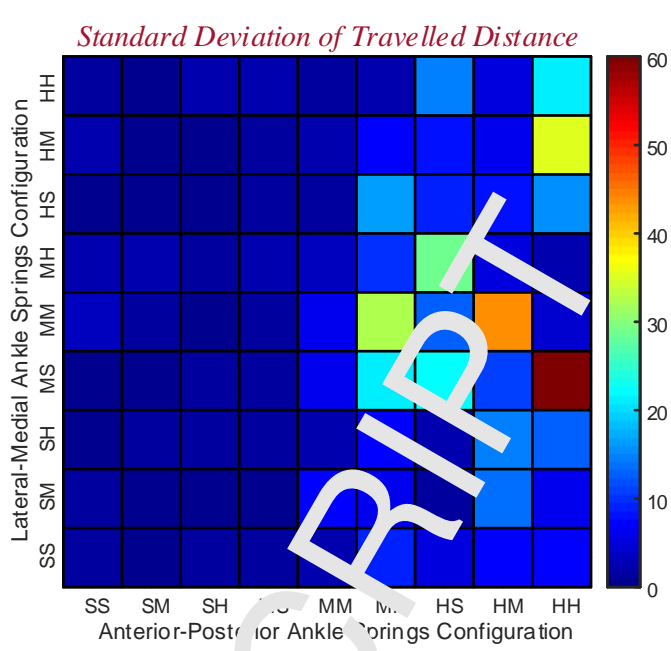

(b)

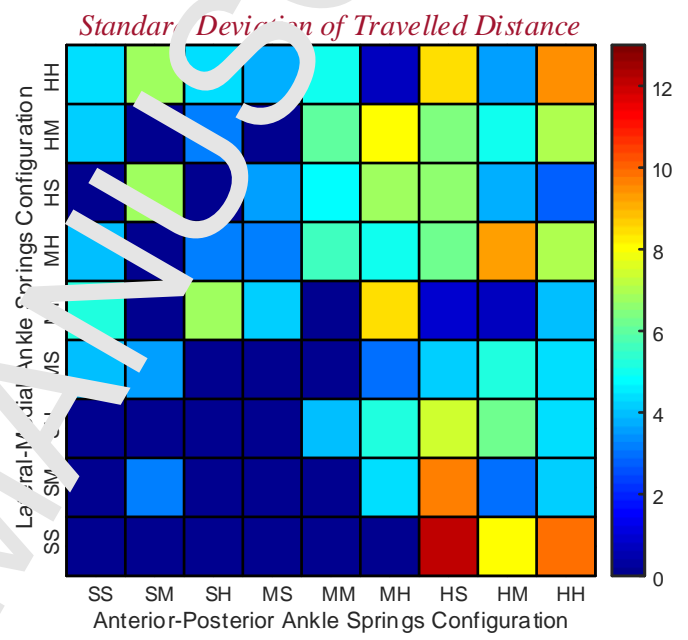

(d)

Figure 6. Travelled distance of short sten Wa. 'ing (a) and long step walking (c) tests in different ankle springs configuration. For each combination, ${ }^{+}$ie $\mathrm{r}$ ean value of distance travelled was calculated using the data from the first 5 successful tests. The stan ${ }^{\text {rd }}$ devi tion of travelled distance for short step (b) and long (d) step walking respectively. S: Soft sprins, M: $N_{\iota}{ }^{d}$ um spring, H: Hard spring.

For short steps test, when setting 'ard springs at the anterior and posterior positions and medium springs at the medial and lat ral osition, the robot walked the full length of the ramp very stably, and it could keep walking farther, : n ever it reached the edge and fell (see Video 1). The maximum distance travelled was $247 \mathrm{~cm}, \mathrm{su}^{\prime}$ cractea .'e foot length $13 \mathrm{~cm}$ from the ramp length $260 \mathrm{~cm}$. For long steps test, with the same configur: ion the naximum average distance was $67.2 \mathrm{~cm}$ much lower than short steps.

The results obtained (st. F" gure 6a and 6c) suggest that once its stiffness overcomes a minimum level, the robot walks ste dier $f_{1}$ " many spring configurations. The main reason to the improvement is the increasing stiffness $i^{f}$ the $s$ rings placed in the sagittal plane, especially the anterior position. They have a main impact $\mathrm{o}$. controling the pitch motion and avoiding that the robot falls forward or backward. The heavy hip desi $i_{i}$ ned to nhance the pendulum motion during walking also makes the robot tend to fall when the stiffness of ae ankle is insufficient. In all the successful tests, the robot can travel more than 4 steps and $t_{1}, s_{1}-3$ configurations set at least one hard spring of $6670 \mathrm{~N} / \mathrm{m}$ which tremendously increases the $\mathrm{s}_{i}$ fness of the ankle in its plane.

Results from short and long steps led to the same conclusion. The test with stiffer spring in the anterior position and soft spring in the posterior position performed successful results, however the case with stiffer spring in the posterior position and soft spring in the anterior position obtained much worse results suggesting that setting stiffer springs in the anterior position can fix instability problems but not the reverse. 


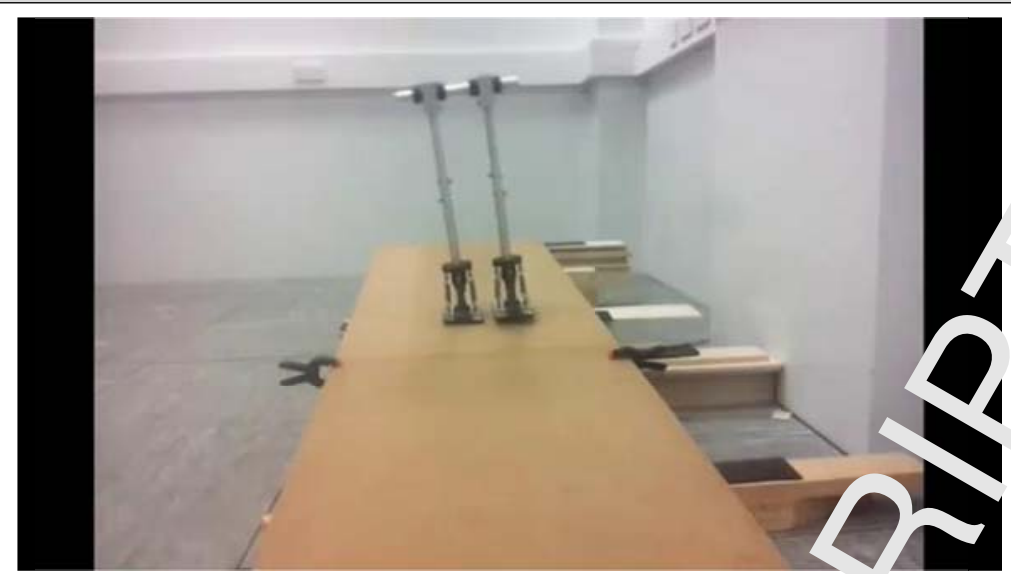

Video 1. Successful walking of short steps test with hard springs at the anterior and posterior positions and medium springs at the medial and lateral position.

For the short steps, setting hard spring in the lateral or medis position produced high stiffness in the coronal plane of the ankle leading to a problem called edge trea ${ }^{\prime} i$.., th 2 raising foot after a very slight inclination from the support leg. Setting medium spring in tis late and medial position facilitated a decent average of $245 \mathrm{~cm}$ travelled distance (see Figure 6a), 5 time more than hard spring. In fact, this difference also occurred in all the testing configured with $\mathrm{h}$ - $\mathrm{d}$ ring of one or two positions in the coronal plane. The average distance travelled for setting at in st one hard spring in the coronal plane is $36.23 \mathrm{~cm}$ (calculated except the trial of which the nu. wer or steps is less than 4). As can be seen in Figure $6 \mathrm{a}$, the best configuration is a symmetric setting wa two medium springs in the coronal plane, followed closely by a configuration with a hard sp ing a the lateral position and a soft spring at the medial position. Setting stiffer springs at the med $\curvearrowright 1$ pos ion than at lateral led to instability, while testing with two soft springs in the coronal plane obtained ho vorst results.

For the case of long step walking, the be - acul were achieved by two configurations, one with a medium lateral spring and a soft medial spring, and the other with two medium springs in the coronal plane (see Figure 6c). The former provider a softer ankle joint stiffness than the latter which enhanced longer steps by performing longer roll $\mathrm{r}$ otion 、 vcle that should match a larger swinging of the opposite leg. In other words, setting stiffer $\operatorname{sprin}_{\zeta}$ at $t^{\prime}$.e lateral position than in the medial one improved the walking stability.

\subsubsection{Step Length and Velocity.}

Comparison between short, $\mathrm{tt}_{\mathrm{r}}$, and long step was analyzed by calculating the average of the most successful short step test and the averaye of the most successful long step test (see Supplementary Table S1). The short steps test act eve 7.8 times more steps than the long steps test. The distance travelled is also 3.7 times longer than the ng step. The step length is increased in $107.8 \%$ for the long step leading to a velocity which is $19, \%$ 'igher than for short steps.

The configuration $w$.h sard springs in the sagittal plane and medium springs in the coronal plane was used to study the $\mathrm{re}^{1}$... entire stable spectr $m$ of $s t p$ lengths. The robot was launched 8 times, each one with a different step length from 3.15 to $5 \mathrm{cr}$. leading to different velocities and number of steps taken (full data can be found in Suppl menta v Table S2). Figures 7a and 7d show the relationships between velocity and step length, velocity and nı nber of steps respectively at the optimal ankle spring setting. We can see that velocity generally mcreases with increased step length. Whereas, the number of steps decreases dramatically ' $h$ ' $\mathrm{n}$ walking velocity increases indicating poor stability at high speed. It can be seen from Figure $7 \mathrm{a}$ that $\mathrm{l} \mathrm{t}$ is difficult for the robot to achieve stable walking when the step length exceeds $9.50 \mathrm{~cm}$.

Figures $7 \mathrm{~b}$ and $7 \mathrm{c}$ show the average velocities at different ankle spring combinations for the short step and long step tests respectively. Overall, the setting of the anterior-posterior springs has larger effect on walking velocity than the medial-lateral springs. The robot tends to walk slowly with soft anteriorposterior springs. For short step walking, the highest velocity $9.63 \mathrm{~cm} / \mathrm{s}$ is achieved at spring setting of anterior medium, posterior hard, lateral medium and medial soft. Whereas, for long step walking, the highest velocity $17.10 \mathrm{~cm} / \mathrm{s}$ is found at spring setting of anterior medium, posterior medium, lateral hard 
and medial hard. Figures $7 \mathrm{e}$ and $7 \mathrm{f}$ show the average velocities at different toe spring combinations for the short step and long step tests respectively. Both medial and lateral toe spring configurations have significant effect on walking velocity. For short step tests, the highest speed $8.81 \mathrm{~cm} / \mathrm{s}$ occurs when both toes are in free moving (no springs). For long step walking, the highest velocity $14.25 \mathrm{~cm} / \mathrm{s}$ is obtained when both toes use hard springs.

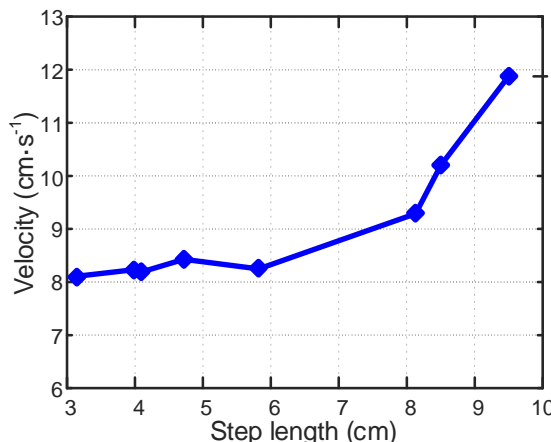

(a)

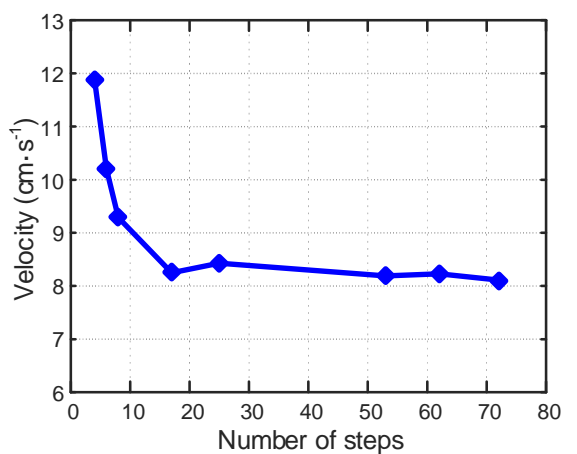

(d)

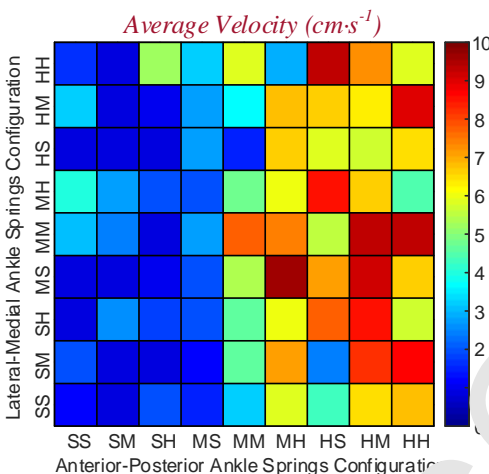

(b)

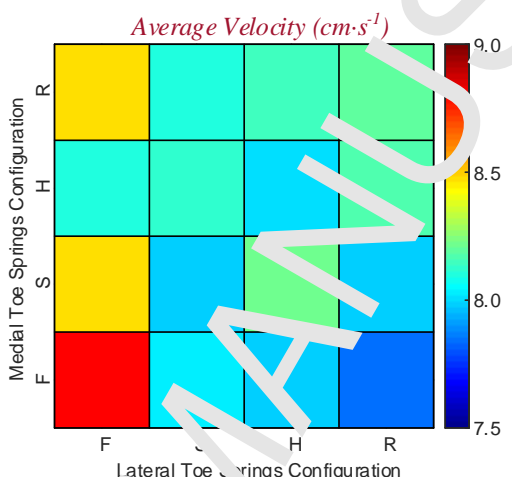

in

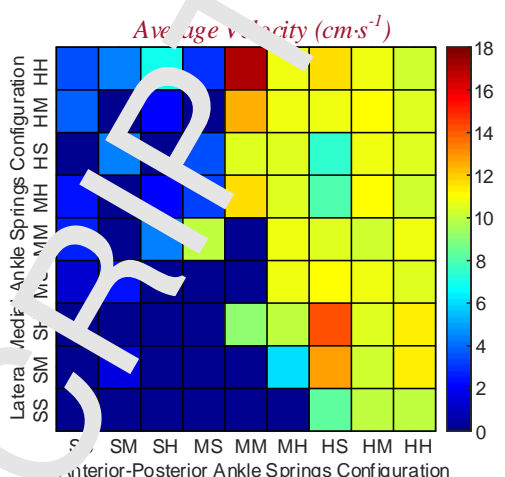

(c)

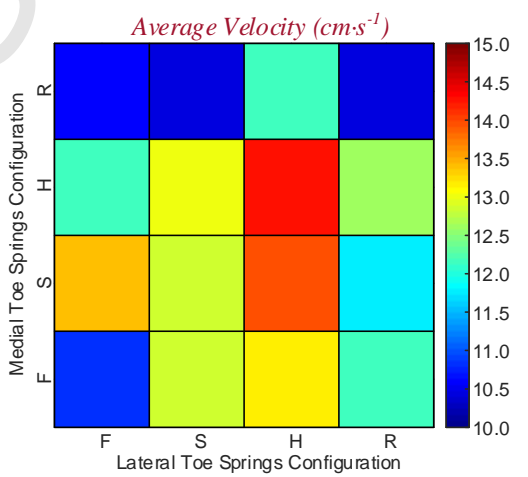

(f)

Figure 7. Relationships among velocity, sten length, number of steps. (a) Velocity vs. step length at the optimal setting of the ankle springs. The veras velocities at different ankle spring combinations for short step (b) and long step (c) tests. (d) Veloci, vs. nul iber of steps at the optimal setting of the ankle springs. The average velocities at different toe spri $\mathrm{g}$ con ${ }^{-} i$ ations for short step (e) and long step (f) tests. For ankle spring setting, S: Soft spring, M: Me tum spring, H: Hard spring. For toe spring setting, F: Free moving (no spring), S: Soft spring, H: Hard spring, ᄀ Rig d connection.

\subsection{Toe test}

The toes were tested $\mathrm{se}^{\mathrm{t}^{-}} \mathrm{r}$ all the configurations from the softest to the stiffest with the most successful setup of the ank 1 . spr $^{\text {ng }}$ system which has hard springs in the anterior and posterior position and medium springs in th $>$ latt. -1 and medial position. There are 4 different settings for each toe, which makes a total of $16 \operatorname{con}^{f}$ gur tions to be tested for short and long motions, raising the total of 32 testing combinations (each coms: ${ }^{\prime} t^{\prime}$ is launched 5 times).

The process $\mathrm{W}^{\prime} \mathrm{KS}$ exac.ly in the same way as the ankle springs test. The detailed testing measurements with he enti : launches can be found in Supplementary Table S3. The standard deviation of distance travellad ... thort steps (Figure 8b) and long steps (Figure 8d) walking in different toe settings were cr mpute to support the rationality of the following analysis.

For short stc, 's wal' ing, the results showed similar travelled distance with an aleatory distribution for all the conf, mations (see Figure 8a), which suggest that the toes have almost no effect on the walking stability. The $w$ sage number of steps is 59.97 with a step length of $3.98 \mathrm{~cm}$, velocity of $8.12 \mathrm{~cm} / \mathrm{s}$ and distance travell ${ }^{1}$ of $236.48 \mathrm{~cm}$. The robot could perform very stable walking motion for short steps independently of the toes stiffness but with the normal falling or deceleration.

For long steps walking, the average distance travelled is $55.73 \mathrm{~cm}$ with a step length of $8.43 \mathrm{~cm}$. The velocity increases from $4.13 \mathrm{~cm} / \mathrm{s}$ up to $12.25 \mathrm{~cm} / \mathrm{s}$ in comparison with short step test, however, taking longer steps entails a great loss of stability decreasing the maximum distance travelled. The robot gets accelerated after 3 steps as the ankles springs cannot control its motion. On the other hand, while the short step test achieves very similar number of steps for every configuration, the results for long steps 
present larger variations depending on the toes setting. The toes stiffness influences slightly to the long steps walking, and the robot can travel $18.8 \mathrm{~cm}$ longer, which supposes an increment of $40.2 \%$. All configurations that set the steel plate blocking a toe (rigid joint) or allowing free motion of the toe obtained worse results (see Figure 8c). The proper setting of the toes stabilized the robot to walk more 10 to $20 \mathrm{~cm}$ distance before falling.

Since the main differences were obtained from cases that blocked at least sne ne or allow free motion for both toes, the results were grouped in function of these toes settings. $\cdots \sim$ first group included all the tests blocking the medial toe (rigid joint), the second group blocking the ater I toe, the third group allowing free motion of the toes, and the rest.

Blocking or allowing free motion of the medial toes entailed worse stahilıy 'see Figure 8c). When blocking the medial toe with a steel plate, the robot had more difficulties ${ }^{+}, \mathrm{cr}+\cdots$ sufficient clearance for the swinging of the leg. Also, blocking the medial toe made the fou $r$ thave like a unique larger

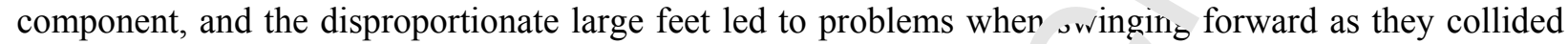
with the ground. Indeed, humans with very large foot may have th: same roblems even if they have knees. On the other hand, blocking the lateral toe did not have the san $n r r$ slem as it can be raised very

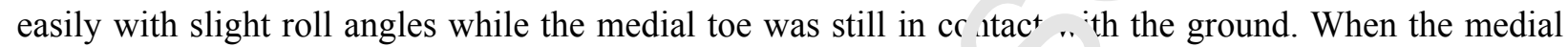
toe was not blocked, the small roll motion required to allow the - wing ng of the legs facilitated higher stability with the same step length. Therefore, this configurai $n$ ootained better results reaching an average of $55.35 \mathrm{~cm}$ distance travelled against $49.95 \mathrm{~cm}$ obtrined by :ettings with the medial toe blocked.

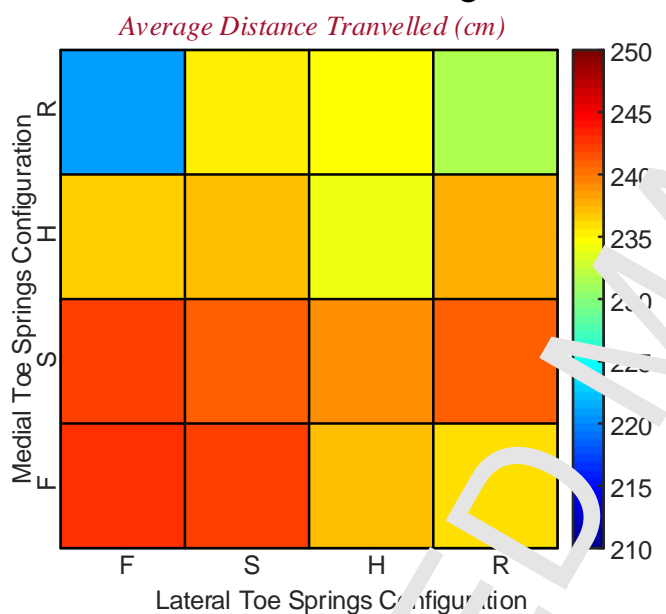

(a)

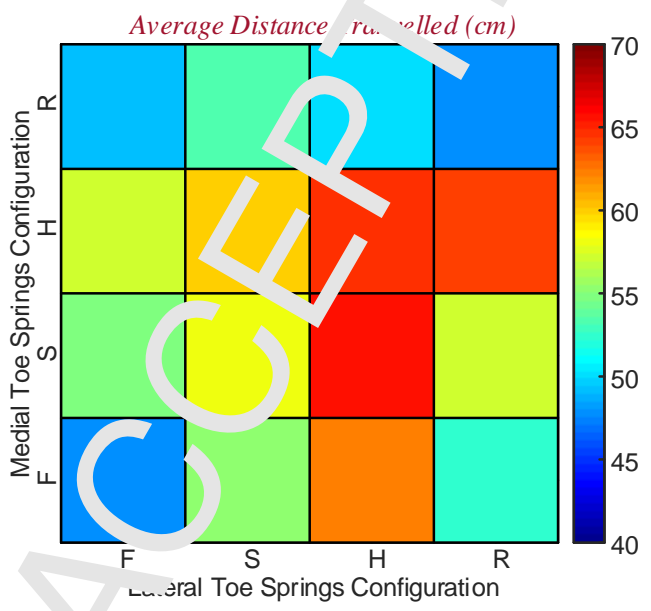

(c)

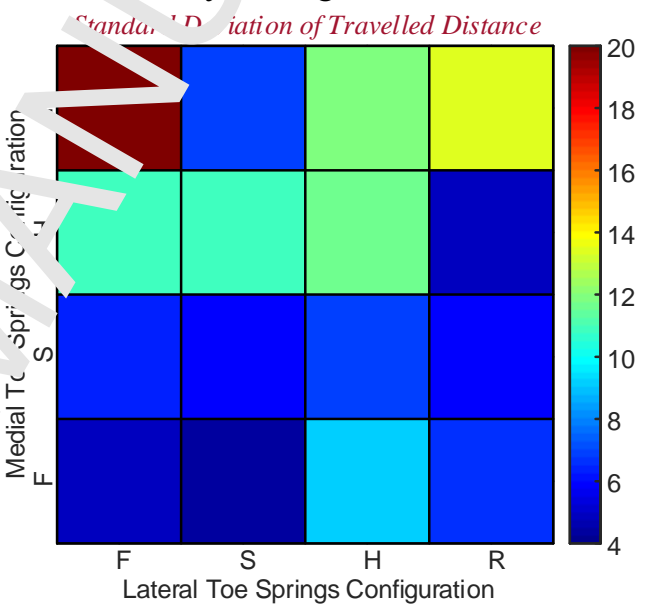

(b)

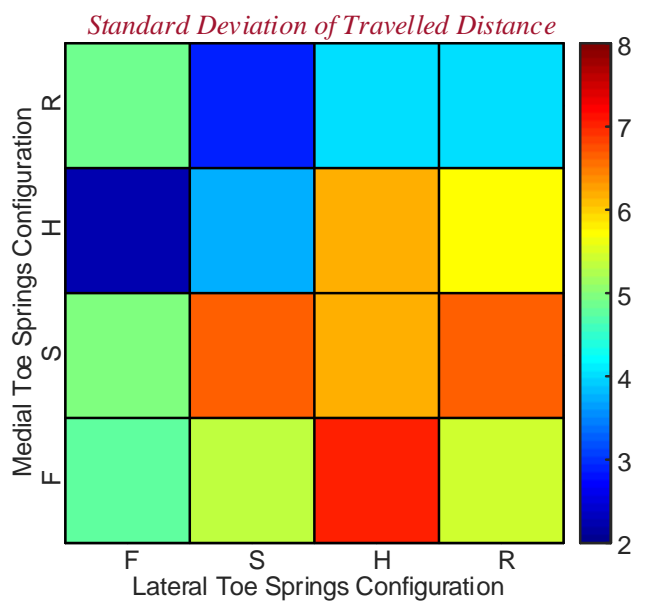

(d)

Figure 8. Travelled distance of short step walking (a) and long step walking (c) in different toes configuration. For each combination, the mean value of distance travelled is calculated using the data from the first 5 successful tests. The standard deviation of travelled distance for short step (b) and long step (d) walking respectively. F: Free moving (no spring), S: Soft spring, H: Hard spring, R: Rigid connection.

For test that allowed free motion of the toes, the results obtained were worse (see Figure 8c). When the robot took longer steps with larger roll or swinging motions, the toes stiffness could help to maintain 
the stability with more distance travelled. It was observed during testing that when the toes rotated freely about their hinges, the robot lost stability sooner when any of its motions went out of its control limits.

The best results were obtained with medium (soft) and stiffer (hard) rubbers. They allowed the bending of the toes during the raising of the foot and enhanced the stability when the robot started losing the stable motion cycle by providing an extra support.

\section{Discussion}

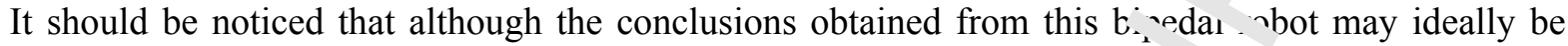
compared with human walking features, it could lead to error for some - nects 's both perform very different walking motions. The fact that humans have knees makes their ' alki is notion very different to imitate. The robot needs roll motion to create clearance that permits the, s to swing forward without colliding with the ground, however, humans do not need such a roll notion as they create clearance by the knee joint. Furthermore, the foot motion is also different as for he robo they land and raise almost parallel with the ramp, while for the humans there is a relative nng _ . th the ground performing a different motion and bending the toes about their joint.

There are 2 main areas of interest to be analyzed in the test ol unis $r$ ssive dynamic walker, the ankle spring system and the toes influence on the walking motion. The robot was tested throughout 810 launches for different ankle spring combinations and 160 for thes set ings separately.

\subsection{Ankle}

For the short step testing, the most successful $\mathrm{sp}_{1} \cdot \sigma$ configuration achieved very good walking stability obtaining an average of $245 \mathrm{~cm}$ travelled : long step test the robot turned much more difficult to $c$ itrol obtaining only $67.2 \mathrm{~cm}$ distance. The robot got worse stability along with increasing step - nth ?nd velocity, especially when the step length overcame $8 \mathrm{~cm}$. The comparison between the mos sucessful results of the short and long step test indicates that the stability of the short steps $w^{\prime}$ ' $^{\prime} m_{5}$ _. much higher achieving 7.8 times more steps and 3.7 times more distance travelled than the longer sto, walking. The step length is increased in $107.8 \%$ for the long step test leading to a $19.3 \%$ hig' $^{\prime}$ w relocity. The robot achieved higher velocity taking longer steps but entailing a much higher inst sility tr mendously reducing the number of steps and distance travelled until failure.

The ankle springs have a main inpr it on the successful of the walking motion. The results suggest that the anterior spring, over any $\mathrm{c}^{t} \mathrm{~h} \mathrm{t}_{\text {. }} \cdot \mathrm{osi}^{+}$on, plays a main role providing stability by holding the robot in the upright position and avoir ng it froın falling forward or backward. It is intuitive thinking that the anterior spring immensely impacts on 'nlding the robot in upright position when walking down the slope, as it tries to recover its free $1 f_{1} \mathrm{~g}_{\mathrm{i}}$ producing a torque about the ankle which avoids the robot from falling forward. On the other hanc, the spring from the posterior position produces a similar torque but in the opposite direction pushin, the r'at to fall. That is why the entire configurations set the anterior spring with longer initial elons atio , i.e, high preload, increasing its force to balance the effect of the posterior spring holding the walker is. "in ight position.

Setting the hard spring. of $6670 \mathrm{~N} / \mathrm{m}$ in the sagittal plane led to the best results, and the springs from the sagittal plane al: $>$ contr ,led the pitch angle of the robot which affected its velocity and step length. They determiner ine posıuon of the massed hips which modified the torques about the ankle joint making the robot walk faster 1 - slower. Successful pitch angles are close to zero. When setting slight larger positive angles, is $\mathrm{rr}^{\prime}$,ot tended to walk faster but leading to higher instability. On the reverse, zero or small negat. ' $c$. $^{-1}$ as made the robot walk slower and take shorter steps. For extreme pitch angles, the robot tended te all forward or backward depending on the sign of the inclination. Furthermore, it is very important to plac 2 each hip-leg component with the exactly same pitch angle so that both legs have the same velocity and step length, as any slight discordance of their pitch angle would make one leg swing faster than the other resulting in longer steps and leading to instability and failure.

The roll motion was capitally controlled by the springs from the coronal plane, and a symmetric setting with two medium springs of $3260 \mathrm{~N} / \mathrm{m}$ attained the highest average travelled distance with great stability, controlled, accurate and repetitive roll motion. Setting stiffer springs at the lateral position than in the medial one led to better results as it enhanced the control of the roll motion by the compression of 
the lateral spring acting as a fixed overtravel stop to limit the maximum roll angle and improve the stability. On the other hand, setting the stiffest spring of $6670 \mathrm{~N} / \mathrm{m}$ in the coronal plane overcame the effect of any other spring placed within its same plane and produced extremely high stiffness leading to problems such as edge tread and instable walking motion. Thus, while the hard spring is beneficial in the sagittal plane to avoid the falling forward, it has a negative effect on the roll motio . when placed in the coronal plane.

The fact that the best configuration of the ankle joint has stiffer stiffness in $\mathrm{t}^{2}$. agittal plane than the coronal plane can be compared in nature with humans where Tibialis Anterior, 'ole' s and Gastrocnemius muscles are much stronger than other muscles around the ankle.

(a)
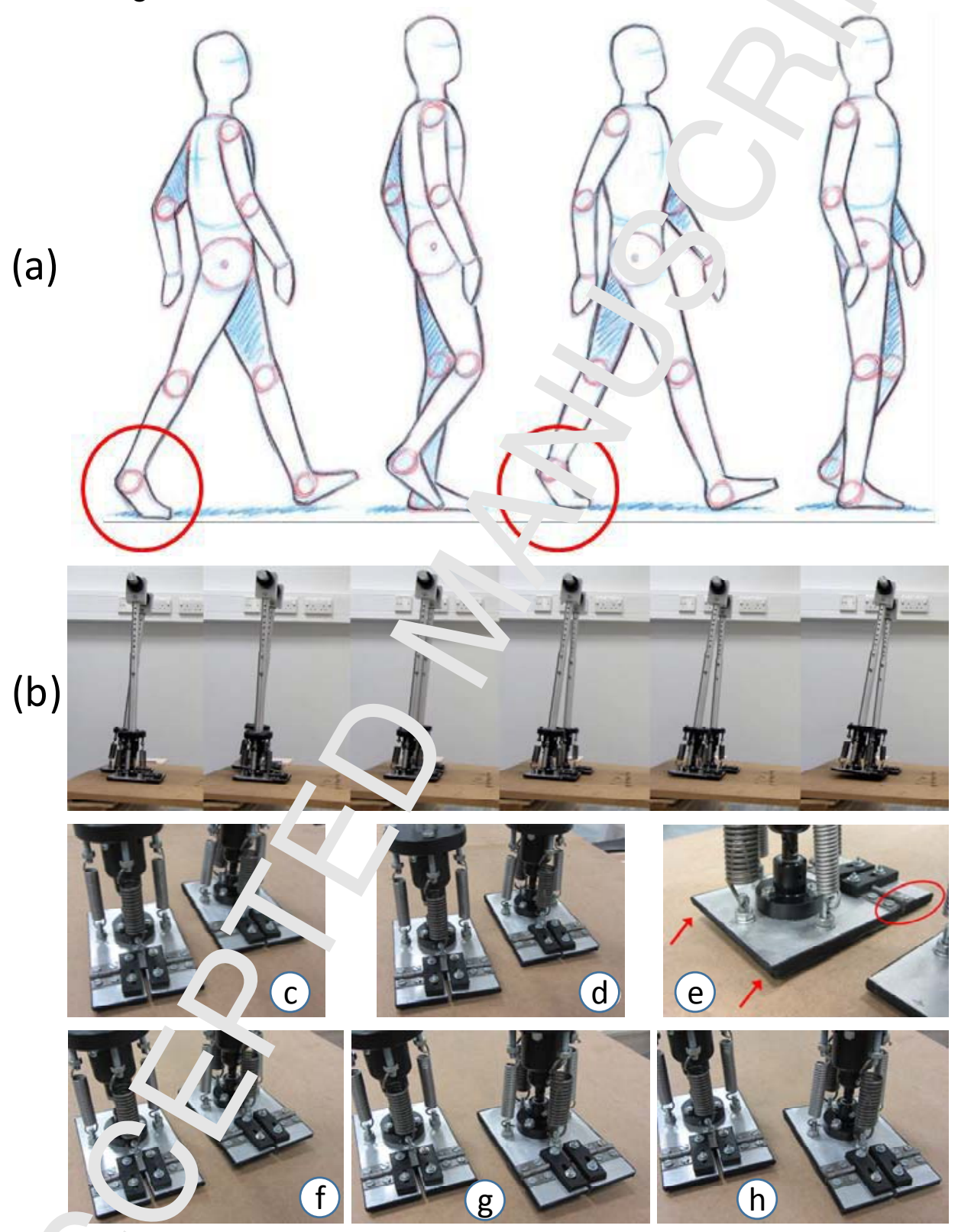

Figure 9. Huma and ro ot walking sequence. (a) Human walks on level ground, where the toes get bended about their joints, rrom push-off to toe-off until initial swing phase. (b) Robot walks on a slight ramp (5.6degree tiltec . . . . to the straight legs without knees, it needs to produce roll motion in order to create sufficient clea $r$ ie that allows the swinging of the leg, which is obtained by bending the knees during human walking. This fe, 'ure leads to a very different walking motion and that the robot toes are bended very slightly in comparison with the human ones. (c) The robot in the upright position with 0-degree roll angle, starts getting tilted raising the left foot of the swinging leg. It can be appreciated from (d) and (e) that the anterior edge is raised as well as the lateral edge of the foot; however the medial toe still touches the ramp. After this instant, the whole foot is raised (f) and swings forward (g). (h) Landing of the left foot which is almost parallel to the ramp. 
The walking motion performed by the robot when taking short or long steps is slightly different changing the toes influence to their respective results. For short steps, the robot performed much smaller roll motion and swinging of the leg, moreover it was slightly tilted backward to reduce torque about the ankle joint produced by the hip weight which would increase step length, velocity, and acceleration. For long steps, its roll motion was larger as well as the swinging of the leg, and the hips were placed slightly forward to increase the trend to walk down the slope with higher velocity ar $\downarrow$ lu rer steps. These differences in the walking motion make the toes have a different level of impact $r$ - he walking stability.

For human walking motion (see Figure 9a), the toes get bended about $t^{\prime}$ eir sint during walking, especially when the support leg gets behind the body. The robot has a differant $w^{-1}{ }^{1}$ king motion. Due to the straight legs without knees, it needs to produce roll motion in order to raice $1 \mathrm{~s}$ ' egs to create sufficient clearance that allows the swinging of the leg. For humans, this roll roti is not needed, and the clearance is created by bending the knees. This feature leads to a very din ${ }^{\circ}$ r $n$ nt walking motion and that the robot toes are bended very slightly in comparison with the human $r$ ass.

Figure $9 \mathrm{~b}$ showed that the support foot was raised by the roll mo on and he toes were barely bended in the robot. For human walking, the support leg stays behind th cringing one for longer time, increasing their relative angle and forcing the toes to get bended, ust $^{2}$. . 7 re raising its foot. Therefore, as longer is the step length, larger is the relative angle between the ..gs ir ireasing the bending of the toes and the impact of their stiffness in the walking motion.

Due to roll motion performed, the foot does not raise $\mathrm{p}$ rallel to he ramp making that the medial toe remains in contact and slightly bended while the rest is lin ${ }^{\prime} a_{1}{ }^{1}$. ushed to move swinging forward (see in Figure 9c to $9 \mathrm{~h}$ and Video 2). This condition makes that the edial toe stiffness impacts on the raising of the foot and swinging motion affecting positively or in ratively to the stability especially for long steps.

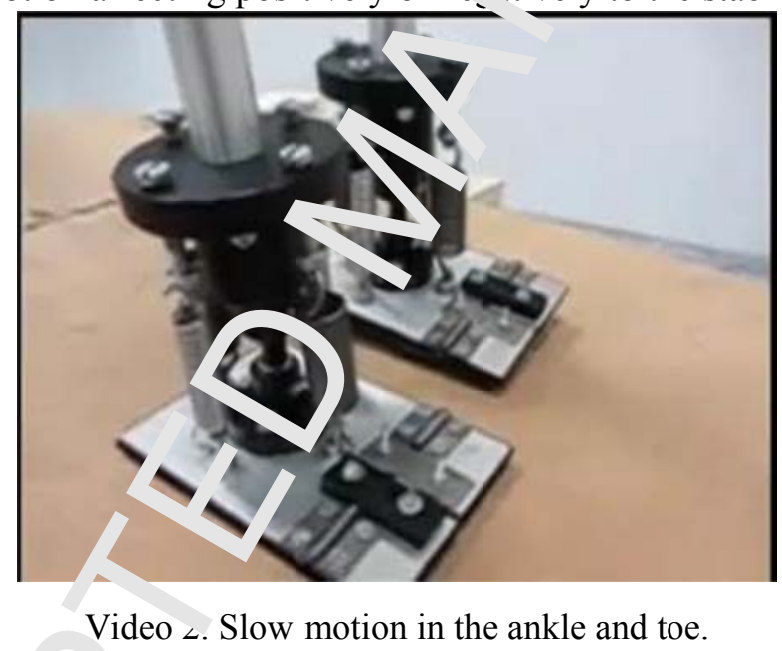

For short step test, the s, $n 1$ ngth is so short that the relative angle between the legs is very small and the supporting leg get $r$ ssed $\left.b c^{-}\right\urcorner r e$ the toes are barely bended not affecting to the walking motion. Therefore, for short ste $s$ th $;$ tof; have no impact on the walking motion and the reasons for failing are the same ones analyzed abu $\sim ;$ the ankle test.

For long steps, $\uparrow$ te toes have a slight impact on the stability of the robot. Blocking the medial toe with the stiffest joint had negat ve effect, and it cannot be bended colliding with the ground and impeding an easy lifting of $t^{2}-$ swinging foot. However, blocking the lateral toe had not such a bad effect, because during the rais ng of $\mathrm{t}$ e support foot the lateral toe was lifted first and higher than the medial one counting with su. results wert sum - - with the stiffest rubber joint (hard spring) at the lateral toe, and any stiffness option except blockit, at the medial toe. Thus, the medial toe could easily be bended to lift the leg and swing forward, and a st.ffer rubber at the lateral position provided more stability when large roll and swinging angles occurred, allowing the robot to travel 10 to $20 \mathrm{~cm}$ more distance before falling apart. However, it should be noticed that the positive effect of stiffer toes when the robot starts losing stability getting accelerated and changing its initial walking motion is moderate. They cannot recover the robot from the falling condition, but just avoid falling sooner which leads to travel more 10 to $20 \mathrm{~cm}$ distance. More control systems should be researched as combining the right toes stiffness with the proper ankle configuration may make the robot walk stably with long steps and even recover the stability when any of 
its motion is out of control.

The fact that the robot has no knees makes the toes influence much lower than expected. The toes permit humans to take longer steps as they remain in contact with the ground providing extra support when the heel has been already raised, which suggests that for knee robots the toes would have a more significant impact in the stability when taking long steps. These statements are ob lously based on the robot dimensions, if the toes or foot are much larger with the same robot heig ${ }^{1}$. , $_{1}$. ir impact would increase disrupting the walking motion.

Concluding, it is encouraged to develop a robot with knees, flat feet, ank e sr ings and toes which can demonstrate and reveal biomechanical principles and natures of legged ${ }^{1} \mathrm{coO}_{1}$. tion of human body. The flat feet facilitate the robot to walk more stably, and a larger contact s, face with the ground increases the friction which can avoid slipping problems and perform a ${ }^{1}$ ette - ralking direction control without bending sideward unintentionally. The ankle springs provide the,$-t$ st the capability of adapting

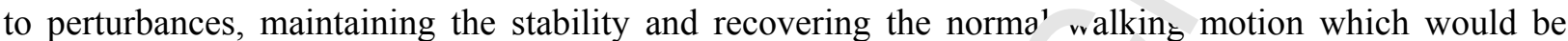
barely possible for a

robot without ankle joint. The toes enhance the stability especially whe tal ng longer steps providing an extra support and helping to recover the stability when any of its mot ${ }^{i}$. goes slightly out of control. The knees would make roll motion unnecessary as the clearance wou.. oe c sated by bending the knee joint. However, this would significantly reduce the complexity of the $\checkmark$ mbıned roll and swinging motions but entail new challenges to control the knee motion.

\section{Acknowledgments}

This study was supported by the Key Project of: 91848204), the National Key R\&D Program of $C_{L_{1}} r_{\perp}$ (No. 2018YFC2001300), and the project of National Natural Science Foundation of China (N, $-16 \%$ 222).

\section{Appendix A. Supplementary dat:}

Supplementary Table S1. Measurem $f$ its o' $\mathrm{ill}$ ankle tests.

Supplementary Table S2. Measuren. nts of ` different step lengths with same ankle and toe settings. Supplementary Table S3. Measur ments ¿' all toe tests.

\section{Reference}

[1] D.A. Winter, Biomechanir , anr motor control of human movement, fourth ed., John Wiley \& Sons, Inc., Hoboken, New Jersey, 20u, 1 .tps://dx.doi.org/10.1002/9780470549148.

[2] A. Cappozzo, Gait Anr ysis Meı Jdology, Hum. Movement Sci. 3(1-2) (1984) 27-50. http://dx.doi.org/10.1' 16/r 167- 457(84)90004-6.

[3] M.G. Pandy, T.P. Andrin rhi Muscle and Joint Function in Human Locomotion, Annu. Rev. Biomed. Eng. 12 ((2010) 401-43־ . nttp://dx.doi.org/10.1146/annurev-bioeng-070909-105259.

[4] L. Ren, R.K. Jon s, D. Hc vard, Whole body inverse dynamics over a complete gait cycle based only on measured kinemat, - J. F omech. 41(12) (2008) 2750-2759. http://dx.doi.org/10.1016/j.jbiomech.2008.06.001.

[5] A. Arndt, P. volf, A . Liu, C. Nester, A. Stacoff, R. Jones, P. Lundgren, A. Lundberg, Intrinsic foot kinematics measured in vivo dur $1 \mathrm{~g}$ the stance phase of slow running, J. Biomech. 40(12) (2007) 2672-2678. http://dx.doi.c $/ 10 ~ J 16 /$ j.jbiomech.2006.12.009.

[6] J. Jeong ' $v$ Oh, C.S. Shin, Measurement of Lower Extremity Kinematics and Kinetics during Valley-Shaped Slope Wa. 'ir s, Int. J. Precis. Eng. Man. 16(13) (2015) 2725-2730. http://dx.doi.org/10.1007/s12541-015-0348y.

[7] Grațiela-Flavia, R.F. Deak, Grosu Emilia, Surface electromyography in biomechanics: Application and signal analysis aspects, Journal of Physical Education \& Sport/Citius Altius Fortius 25(4) (2009) 56-65.

[8] C.J.D. Luca, The use of surface electromyography in biomechanics, J. Appl. Biomech. 13(2) (1997) 135-163. https://dx.doi.org/10.1123/jab.13.2.135.

[9] C.J. De Luca, L.D. Gilmore, M. Kuznetsov, S.H. Roy, Filtering the surface EMG signal: Movement artifact and baseline noise contamination, J. Biomech. 43(8) (2010) 1573-1579. http://dx.doi.org/10.1016/j.jbiomech.2010.01.027.

[10] M. Sartori, M. Reggiani, D. Farina, D.G. Lloyd, EMG-Driven Forward-Dynamic Estimation of Muscle Force 
and Joint Moment about Multiple Degrees of Freedom in the Human Lower Extremity, PLoS One 7(12) (2012) e52618. http://dx.doi.org/10.1371/journal.pone.0052618.

[11] T.S. Buchanan, D.G. Lloyd, K. Manal, T.F. Besier, Estimation of muscle forces and joint moments using a forward-inverse dynamics model, Med. Sci. Sports Exerc. 37(11) (2005) 1911-1916. http://dx.doi.org/10.1249/01.mss.0000176684.24008.6f.

[12] T. Fukunaga, Y. Kawakami, K. Kubo, H. Kanehisa, Muscle and tendon interaction durir s human movements, Exerc. Sport Sci. Rev. 30(3) (2002) 106-110. http://dx.doi.org/10.1097/00003677-200` J7u`-00003.

[13] Y. Kawakami, T. Fukunaga, New insights into in vivo human skeletal muscle function Exerc. sport Sci. Rev. 34(1) (2006) 16-21. http://dx.doi.org/10.1097/00003677-200601000-00005.

[14] S. Pillen, N. van Alfen, Skeletal muscle ultrasound, Neurol. Res. 33(10) (2011) 101, 1,24. http://dx.doi.org/10.1179/1743132811y.0000000010.

[15] L. Ren, D. Howard, L.Q. Ren, C. Nester, L.M. Tian, A generic analytical foot ${ }^{-1}{ }^{1}$ ove translational ankle kinematics in gait simulation studies, J. Biomech. 43(2) ( $\mathrm{J} 10$ ? 1 -202. http://dx.doi.org/10.1016/j.jbiomech.2009.09.027.

[16] M.G. Pandy, Computer modeling and simulation of human movement, A $\cdots$-1. Rt. Biomed. Eng. 3((2001) 245-273. http://dx.doi.org/10.1146/annurev.bioeng.3.1.245.

[17] T.R. Jenkyn, A.C. Nicol, A multi-segment kinematic model of the foot ith a nc rel definition of forefoot motion for use in clinical gait analysis during walking, J. Biomech. $\left.{ }^{\wedge} / 14\right)(2, v /)$ 3271-3278. http://dx.doi.org/10.1016/j.jbiomech.2007.04.008.

[18] P. Holmes, R.J. Full, D. Koditschek, J. Guckenheimer, The dynamı of leg şed locomotion: Models, analyses, and challenges, Siam Rev. 48(2) (2006) 207-304. http://dx.doi.ory'10.1_- //S0036144504445133.

[19] F.C. Anderson, M.G. Pandy, Dynamic optimization of human walking. J. Biomech. Eng. 123(5) (2001) 381390. http://dx.doi.org/10.1115/1.1392310.

[20] Vukobratović M, Stepanenko J, On the stability of anthropon. ‘rohı systems, Math. Biosci. 15(1-2) (1972) 137. https://dx.doi.org/10.1016/0025-5564(72)90061-2.

[21] M. Vukobratović, B. Borovac, Zero-moment point — thir, five years of its life, Int. J. Hum. Robot. 1(01) (2004) 157-173. https://dx.doi.org/10.1142/S021984360400nn83.

[22] M. Hirose, K. Ogawa, Honda humanoid robots devel ' 'me ı, . nilos T R Soc A 365(1850) (2007) 11-19. http://dx.doi.org/10.1098/rsta.2006.1917.

[23] D. Gouaillier, V. Hugel, P. Blazevic, C. Kilner, J. ‘ ‘ cea x, P. Lafourcade, B. Marnier, J. Serre, B. Maisonnier, Mechatronic design of NAO humanoid, . ᄀ: $\angle$ v09 IEEE International Conference on Robotics and Automation, (ICRA), 2009, pp. 769-774. http., ‘x.uvi. g/10.1109/ROBOT.2009.5152516.

[24] A.K. Singh, G.C. Nandi, NAO humanoid robot: An. 'vsis of calibration techniques for robot sketch drawing, Robot. Auton. Syst. 79((2016) 108-121. httn·/dx.doi.org/10.1016/j.robot.2016.01.009.

[25] Nelson G, Saunders A, Neville N, Swill: .tg B, L mndaryk J, Billings D, Lee C, Playter R, Raibert M, Petman: A humanoid robot for testing chemical pl ‘ sctive c othing, J. Robot Soc. Jpn. 30(4) (2012) 372-377. https://dx.doi.org/10.7210/jrsj.30.37?.

[26] Boston Dynamics, Atlas - the agile unth spomorphic robot. https://www.bostondynamics.com/atlas, 2013.

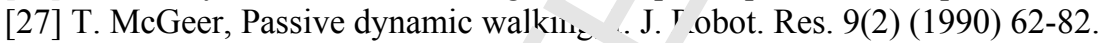
https://dx.doi.org/10.1177\%2F0 .78364y, 00900206.

[28] R. Tedrake, T.W. Zhang, M.F Fo - H.S. Seung, Actuating a simple 3D passive dynamic walker, in: 2004 IEEE International Conference on Rouvtics and Automation, (ICRA), 2004, pp. 4656-4661. http://dx.doi.org/10.1109/P sbor 2004.1302452.

[29] M. Wisse, G. Feliksdal, J. ^n rankenhuyzen, B. Moyer, Passive-based walking robot - Denise, a simple, efficient, and lightweig' i bipeu, 'EEE Robot. Autom. Mag. 14(2) (2007) 52-62. http://dx.doi.org/10.1' J9/A ra.2007.380639.

[30] S.H. Collins, A. Ruina, 1 oipe al walking robot with efficient and human-like gait, in: 2005 IEEE International Conference on Ror _.ics anc sutomation, (ICRA), 2005, pp. 1983-1988. http://dx.doi.org/ 0.1109, 'OBOT.2005.1570404.

[31] R.E. Kearney, I.W Hunte , System identification of human joint dynamics, Crit. Rev. Biomed. Eng. 18(1) (1990) 55-87

[32] R.E. Kearne ', R.B. . ein, L. Parameswaran, Identification of intrinsic and reflex contributions to human ankle stiffness dyn، nics, J .EE Trans. Biomed. Eng. 44(6) (1997) 493-504. http://dx.doi.org/10.1109/10.581944.

[33] H. Lee, D Ho, Mı.r. Rastgaar, H.I. Krebs, N. Hogan, Multivariable static ankle mechanical impedance with relaxed n. 'sc' s, .. Biomech. 44(10) (2011) 1901-1908. http://dx.doi.org/10.1016/j.jbiomech.2011.04.028.

[34] I.D. Loram, M. Lakie, Direct measurement of human ankle stiffness during quiet standing: the intrinsic mechanical st. Ifness is insufficient for stability, J. Physiol. 545(3) (2002) 1041-1053. https://dx.doi.org/10.1113/jphysiol.2002.025049.

[35] H. Lee, N. Hogan, Time-Varying Ankle Mechanical Impedance During Human Locomotion, IEEE Trans. Neural Syst. Rehab. Eng. 23(5) (2015) 755-764. http://dx.doi.org/10.1109/Tnsre.2014.2346927.

[36] E.M. Ficanha, G.A. Ribeiro, M. Rastgaar, Design and Evaluation of a 2-DOF Instrumented Platform for Estimation of the Ankle Mechanical Impedance in the Sagittal and Frontal Planes, IEEE-ASME T. Mech. 21(5) (2016) 2531-2542. http://dx.doi.org/10.1109/Tmech.2016.2552406.

[37] A. Leardini, M.G. Benedetti, L. Berti, D. Bettinelli, R. Nativo, S. Giannini, Rear-foot, mid-foot and fore-foot 
motion during the stance phase of gait, Gait Posture 25(3) (2007) 453-462.

http://dx.doi.org/10.1016/j.gaitpost.2006.05.017.

[38] T. Otani, K. Hashimoto, T. Isomichi, M. Sakaguchi, Y. Kawakami, H.O. Lim, A. Takanishi, Joint Mechanism That Mimics Elastic Characteristics in Human Running, Machines 4(1) (2016).

http://dx.doi.org/10.3390/machines4010005.

[39] T. Otani, K. Hashimoto, T. Isomichi, S. Miyamae, M. Sakaguchi, Y. Kawakami, H. Lim A. Takanishi, Joint Mechanism Coping with Both of Active Pushing-off and Joint Stiffness Based on Hur . In, . - mansy 21 - Robot Design, Dynamics and Control (2016) 243-250. http://dx.doi.org/10.1007/978-3-319-32714-2_<7.

[40] T. Otani, K. Hashimoto, M. Yahara, S. Miyamae, T. Isomichi, M. Sakaguchi, Y. K wak mi, H.O. Lim, A. Takanishi, Running with Lower-Body Robot That Mimics Joint Stiffness of Humaı. i : 2015 IEEE/RSJ International Conference on Intelligent Robots and Systems, (IROS), 2015, pp. 3, ‘9-3y, 1 http://dx.doi.org/10.1109/IROS.2015.7353936.

[41] Y. Ogura, K. Shimomura, H. Kondo, A. Morishima, T. Okubo, S. Momoki, ' ..O. ‥ A. Takanishi, Humanlike walking with knee stretched, heel-contact and toe-off motion by a huma. vir robot, in: 2006 IEEE/RSJ International Conference on Intelligent Robots and Systems, (IROS), $200 \%$ วp. ग, 75-3981. http://dx.doi.org/10.1109/Iros.2006.281834.

[42] K. Hashimoto, Y. Takezaki, K. Hattori, H. Kondo, T. Takashima, H. Li ` A. Ta anishi, A study of function of foot's medial longitudinal arch using biped humanoid robot, in: $2010{ }^{r} \mathrm{E}_{1}=\ldots$ International Conference on Intelligent Robots and Systems, (IROS), 2010, pp. 2206-2211. http //dx we org/10.1109/IROS.2010.5650414.

[43] S. Kajita, K. Kaneko, M. Morisawa, S. Nakaoka, H. Hirukawa, ZN_' Jased siped running enhanced by toe springs, in: 2007 IEEE International Conference on Robotics ana ' 'itolı....on, (ICRA), 2007, pp. 3963-3969. http://dx.doi.org/10.1109/Robot.2007.364087.

[44] K. Kaneko, F. Kanehiro, M. Morisawa, T. Tsuji, K. Miura, S. 1 kaokr, S. Kajita, K. Yokoi, Hardware Improvement of Cybernetic Human HRP-4C for Entertainmc. Use, in: 2011 IEEE/RSJ International Conference on Intelligent Robots and Systems, (IROS), 2011 nn ‘392-4399. http://dx.doi.org/10.1109/IROS.2011.6094415.

[45] K. Miura, M. Morisawa, F. Kanehiro, S. Kajita, K. Kaneko, K. Yokoi, Human-like Walking with Toe Supporting for Humanoids, in: 2011 IEEE/RSJ Intern. tion « onference on Intelligent Robots and Systems, (IROS), 2011, pp. 4428-4435. http://dx.doi.org/10 1109, ‘OS.2011.6094838.

[46] T. Narukawa, K. Yokoyama, M. Takahashi, K. Yc. 'เ' '? L ssign and construction of a simple 3D straightlegged passive walker with flat feet and ankle spring. Journal of System Design and Dynamics 3(1) (2009) 112. http://dx.doi.org/10.1299/jsdd.3.1.

[47] T. Narukawa, K. Yokoyama, M. Takahashi, K. Yos: 'da, A Simple 3D Straight-Legged Passive Walker with Flat Feet and Ankle Springs, in: 2008 IEEF/R SJ International Conference on Intelligent Robots and Systems, (IROS), 2008, pp. 2952-2957. http://dx.' vi.org '0.1109/IROS.2008.4651174.

[48] M. Nordin, V.H. Frankel, Basic biome . 'nics of the musculoskeletal system, fourth ed., Lippincott Williams \& Wilkins, Philadelphia, PA, USA, $20^{1} 2$.

[49] S.H. Collins, M. Wisse, A. Ruina, thr e-dimensional passive-dynamic walking robot with two legs and knees, I. J. Robot. Res. 20(7) (2001) 607-o_ ‘ nttp /dx.doi.org/10.1177/02783640122067561.

[50] S. Collins, A. Ruina, R. Tedrak, M. W1s , Efficient bipedal robots based on passive-dynamic walkers, Science 307(5712) (2005) 108 _-ı 5 . http://dx.doi.org/10.1126/science.1107799. 

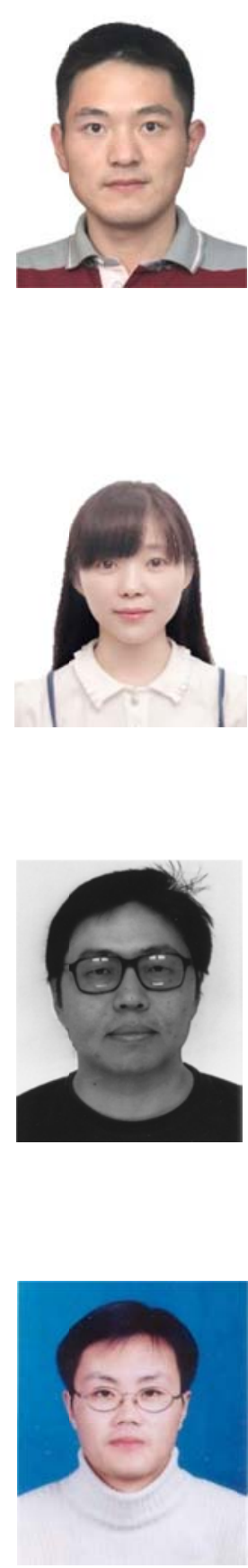

Zhihui Qia 1 re eiveu the $\mathrm{PhD}$ degree in Bionic Science and Technology from Jilin L ir srsit , China in 2010. He won the China Scholarship Council (CSC) sr ،olarsh $\mathrm{i}_{\mathrm{r}}$ and came to King's College London, UK during his $\mathrm{PhD}$ study. Dr. Tian is currently a professor in Key Laboratory of Bionic Engir-aring, Mınistry of Education, and the Deputy Director in Department of sion : and Power Engineering, College of Biological and Agricultural Engı. sing, Jilin University. His research interests include human jior echanics, innovative bionic robotics, and bionic healthcare engineering.

Le Ren received a BSc in Mechanical Engineering from Jilin University, a MSc and first PhD degrees in Vehicle Engineering from National Laboratory of Automotive Dynamic Simulation. He then came to Centre for Rehabilitation and Human Performance Research, University of Salford and received his second PhD in Biomechanics. From 2008 to 2010, he was with King's College London as a lecturer in the Division of Engineering. Dr. Ren is currently a reader in the School of MACE, University of Manchester, and Changjiang Chair Professor in Jilin University. His research interests include human biomechanics, biorobotics, prosthetics, exoskeletons and bioengineering. 


\section{HIGHLIGHTS}

- A three-dimension passive dynamic walker is developed to investigate ankle and toe function in walking

- The stiffness in the ankle must exceed a minimum level to maintain walkino stability

- The ankle joint should have stiffer stiffness in anterior-posterir. pr sition than in medial-lateral position

- $\quad$ Adding toes (medial softer than lateral) may benefit robot’s biped ln romı : $n$ especially for longer step length 